\title{
2 Der Aufstieg des Hauses Ullstein (1877-1932)
}

\subsection{Vorgeschichte und Etablierung auf dem Zeitungsmarkt}

Die Spuren der Familie Ullstein lassen sich bereits früh mit dem Verlagsgewerbe in Verbindung bringen. Unter dem Namen Ullmann lebten sie als Papierhändler in Bayern, 1690 werden sie als Besitzer einer Druckerei in Unterfarrnbach genannt. ${ }^{1} 1727$ verlegten die Ullmanns ihr Geschäft ins nahe Fürth, da sich hier ihr wichtigster Auftraggeber, die Jüdische Hohe Schule, befand. Der Sohn von Moses Ullmann (1748-1829), der Papiergroßhändler Hajum Hirsch Ullmann (17921875) ${ }^{2}$, änderte nach dem Bayerischen Judenedikt von 1813 den Familiennamen: Aus Ullmann wurde 1816 zunächst Uhlstein, dessen Schreibweise später in Ullstein abgeändert wurde. ${ }^{3}$

Hajum Hirsch Ullstein, Inhaber der Papierhandelsfirma H. H. Ullstein, ehelichte Hannah Berlin (1794-1858), die Tochter des Oberlandesrabbiners, Spiegelglas-Fabrikanten und königlich-westfälischen Konsistorialrats Mayer Berlin, Enkelin des ansbachischen Hofmünzlieferanten Berlin und Urenkelin des Rabbinatsbeisitzers Abraham Mayer Berlin. Hajum und Hannah bekamen fünf Kinder: Isaak (1820-1862), Julius (*1823), Sophie (1824-1892), Löb (1826-1899) und Max Wilhelm (1836-1895). ${ }^{4}$ Der heranwachsende Löb Ullstein, benannt nach dem Vorfahren mütterlicherseits Löb Meier Berlin, beschloss später - wohl aus Assimilierungsgründen - seinen Namen in Leopold Ullstein zu ändern. ${ }^{5}$

Die Erzeugung von Druckpapier nahm in Deutschland um die Mitte des 19. Jahrhunderts einen deutlichen Aufschwung, wobei das Schwergewicht der

1 Zur langen und beeindruckenden Geschichte der Ullsteins siehe Ullstein, Frederick: Die Ullsteins - Hundert Jahre später. In: Hundert Jahre Ullstein 1877-1977. Band 1. Hrsg. von Joachim W. Freyburg u. Hans Wallenberg. Frankfurt a. M./Berlin/Wien 1977. S. 35-46, hier S. 36.

2 Mehr zur Genealogie sowie zur Familiengeschichte insgesamt bei Laabs, Rainer: Eintrag „Ullstein, Verlegerfamilie“. In: Neue Deutsche Biographie. Hrsg. von der Historischen Kommission bei der Bayerischen Akademie der Wissenschaften. Band 26. Berlin 2016. S. 575-578, hier S. 575 f.

3 Vgl. Ried, Claudia: Die bayerische Judenpolitik in der ersten Hälfte des 19. Jahrhunderts und deren Folgen für das schwäbische Landjudentum. In: Aschkenas - Zeitschrift für Geschichte und Kultur der Juden 21 (2011). Heft 1-2. S. 79-104.

4 Siehe Titel, Volker: Bürgersinn und jüdische Lebenswelt. Ullsteins Fürther Wurzeln. In: „Der ganze Verlag ist einfach eine Bonbonniere“. Ullstein in der ersten Hälfte des 20. Jahrhunderts. Hrsg. von David Oels u. Ute Schneider. Berlin/München/Boston 2015 (Archiv für Geschichte des Buchwesens 10). S. 365-387, hier S. 366.

5 Zur Namensänderung: Nadolny, Sten: „Frau Ullstein“ - Des Imperiums weibliche Linie. In: 125 Jahre Ullstein. Presse- und Verlagsgeschichte im Zeichen der Eule. Hrsg. vom Axel Springer Verlag. Berlin 2002. S. 28-33, hier S. 28.

๖ Open Access. () 2020 Juilane Berndt, publiziert von De Gruyter. (๕) By Dieses Werk ist lizenziert unter der Creative Commons Attribution-NonCommercial-NoDerivatives 4.0 License.

https://doi.org/10.1515/9783110630503-002 
deutschen Papiererzeugung in Sachsen, Schlesien und Bayern lag. ${ }^{6}$ Die Vossische Zeitung etwa, wie die meisten führenden Publikationen in Berlin ansässig, bezog ihr Papier von der Großhandelsfirma H. H. Ullstein in Fürth. Als sich Hajum Hirsch Ullstein 1847 in den Ruhestand begab, übergab er das Familienunternehmen an seine Söhne. Die Brüder verlegten den Sitz der Firma nach Leipzig, das sich zur Hauptstadt des deutschen Verlagsgewerbes aufgeschwungen hatte. Doch nach Streitigkeiten, vor allem mit seinem Bruder Julius, holte sich Leopold Ullstein die Erlaubnis seines Vaters ein, das Familienunternehmen zu verlassen und sich selbstständig zu machen. Im Revolutionsjahr 1848 eröffnete Leopold im Alter von 22 Jahren seine eigene Papiergroßhandlung - in Berlin. Zunächst bezog er ein Kontor in der Brüderstraße in der Nähe des Kupfergrabens, dann zog er mit seinem Geschäft in die Friedrichsgracht, bis er schließlich Geschäft und Wohnung in die Wilhelmstraße 71 verlegte. ${ }^{7}$

Die anbrechende zweite Hälfte des 19. Jahrhunderts, markiert durch die Revolution 1848, war für das deutsche Judentum eine Periode der wirtschaftlichen Neuentfaltung auf vielen Gebieten. Nicht in Zünften oder Ständen der vormodernen Welt eingebunden, entwickelte das Judentum vor dem Hintergrund der neuen politischen Freiheit einen bürgerlichen Geist, der zur Erfolgsgeschichte zahlreicher aufstiegsorientierter Individuen gedeihen sollte. ${ }^{8}$

Leopold Ullstein nun interessierte sich nicht nur für Papier, sondern auch für das, was auf ihm gedruckt wurde. So gehörten bald nach seiner Ankunft in Berlin wichtige Namen der publizistischen Landschaft nicht nur zu seinem Kunden-, sondern auch zu seinem Freundeskreis: etwa Albert Hofmann vom Kladderadatsch, Ludwig von Schaeffer-Voit vom Bazar oder Gustav Hempel, der spätere Herausgeber der Täglichen Rundschau. ${ }^{9}$ Für den aufmerksamen Zeitgenossen gab es jetzt zahlreiche Vorboten für die bevorstehende „Zeitungs-Explosion“, die sich zwischen der Reichsgründung und dem Ersten Weltkrieg ereignen sollte: die stark beschleunigte industrielle Entwicklung, die Gewerbefreiheit, die durch die Landflucht bedingten zahlreichen neuen Arbeitskräfte in den Großstädten, die neuen Verkehrsmittel für einen umfangreichen Vertrieb, die neuen Kommunikationsmittel für die rasche Nachrichtenbeschaffung und

\footnotetext{
6 Siehe Mendelssohn, Zeitungsstadt, S. $100 \mathrm{f}$.

7 Dazu Mendelssohn, Zeitungsstadt, S. $100 \mathrm{f}$.

8 Vgl. hierzu: Stölzl, Christoph: Der Ullstein-Geist. Katalysator gesellschaftlicher Modernisierung. In: 125 Jahre Ullstein. Presse- und Verlagsgeschichte im Zeichen der Eule. Hrsg. vom Axel Springer Verlag. Berlin 2002. S. 8-13, hier S. 9.
}

9 Mendelssohn, Zeitungsstadt, S. 102. 
das durch die freie Konkurrenz bedingte starke Ansteigen der Geschäftsanzeigen. ${ }^{10}$

Das preußische Pressegesetz vom 12. Mai 1851 galt zwar als repressiv, wurde jedoch in der Folgezeit durch konkretisierende Ergänzungen abgemildert. ${ }^{11}$ Bis 1848 hatte es in Berlin drei Tageszeitungen bei 400.000 Einwohnern gegeben; 1860, bei 500.000 Einwohnern, war die Anzahl der täglichen Publikationen bereits auf sieben angestiegen; sechs Jahre später wies Berlin 700.000 Einwohner und zehn Tageszeitungen auf, bei Reichsgründung und 930.000 Einwohnern waren es bereits zwölf Tageszeitungen.

Neben seinem erwachenden publizistischen Interesse engagierte sich der gut vernetzte Ullstein im nachmärzlichen Berlin nun immer stärker im linksliberalen Spektrum der Kommunalpolitik, etwa zusammen mit Rudolf Virchow für Sozial- und Gesundheitsreformen. ${ }^{12}$ Ullsteins politische Interessen und publizistische Neigungen gingen in der preußischen Hauptstadt ohnehin Hand in Hand: Die im Juni 1861 gegründete Deutsche Fortschrittspartei (DFP) genoss mit ihrer Ausrichtung das Wohlwollen der zu dieser Zeit drei auflagenstärksten Blätter Berlins, nämlich der Volks-Zeitung (22.000 verkaufte Exemplare), der Königlich privilegirten Berlinischen Zeitung (13.000 Exemplare) sowie der NationalZeitung (8.500 Exemplare). ${ }^{13}$

Im Jahr 1857 ehelichte Leopold Ullstein Mathilda Berend, die Tochter eines Zahnarztes aus Manchester. Wie die Ullsteins waren die Berends sephardische Juden, deren Vorfahren aus Südeuropa stammten. ${ }^{14}$ In vierzehnjähriger Ehe bekamen Leopold und Mathilda sieben Kinder: die Söhne Hans (1859-1935), Louis Ferdinand (1863-1933), benannt nach dem preußischen Rebellenprinzen, und Franz (1868-1945) sowie die Töchter Käthe (1860-1931), Else (1862-1959), Alice (1866-1938) und Mathilde (1871-1933). Nach dem Tod seiner Gattin Mathilda heiratete Leopold ein weiteres Mal, Elise Pintus (1850-1923), die Tochter eines Magdeburger Kaufmanns. Auch mit ihr bekam er Kinder: Rudolf (1874-1964), Hermann (1875-1943) und Antoni, genannt Toni (1877-1946). ${ }^{15}$ Die fünf Söhne Hans, Louis, Franz, Rudolf und Hermann sollten - je nach ihren Geschicken und Neigungen - in den kommenden Jahrzehnten das Ullstein-Imperium nicht

10 Mendelssohn, Peter de: Die Anfänge. In: Hundert Jahre Ullstein 1877-1977. Band 1. Hrsg. von Joachim W. Freyburg u. Hans Wallenberg. Frankfurt a. M./Berlin/Wien 1977. S. 47-82, hier S. $54 \mathrm{f}$.

11 Nach Koszyk, Kurt: Deutsche Presse im 19. Jahrhundert. Geschichte der deutschen Presse, Teil II. Berlin 1966 (Abhandlungen und Materialien zur Publizistik 6), S. 122.

12 Vgl. Stölzl, Ullstein-Geist, S. 9.

13 Hierzu Koszyk, 19. Jahrhundert, S. 143.

14 Siehe Nadolny, Frau Ullstein, S. 28.

15 Vgl. Nadolny, Sten: Ullsteinroman. Berlin 2004, S. 486 f. 
nur führen, sondern entscheidend neu ausrichten und so die deutsche Presselandschaft bis zur Zäsur 1933 nachhaltig beeinflussen.

Für Leopold Ullstein war 1871 nicht nur das Jahr, in dem er Witwer wurde und sich wiederverheiratete, es war auch das Jahr, in dem man ihn für die „Freisinnigen“ in die Berliner Stadtverordnetenversammlung wählte. Hier, im „Roten Haus“, regte er in den kommenden Jahren soziale und kommunale Reformen an. Ullsteins Laufbahn als Kommunalpolitiker fand mit seiner Niederlage bei den Erneuerungswahlen 1877 ein unerwartetes Ende.

Doch der umtriebige Unternehmer hatte ohnehin bereits Pläne für eine ganz andere Bühne als die Politik. Am 15. Juli 1877 schrieb Leopold an seine Tochter Käthe:

Gestern habe ich nun in der That und wirklich einen großen Kauf gethan, nämlich eine Zeitung nebst Buchdruckerei; ich glaube dadurch für Hans und Louis gesorgt zu haben. Hans kann einst die Redaction übernehmen, Louis soll Buchdrucker, wie ich es immer vorhatte, werden; inzwischen habe ich aber die Arbeit, es ist aber eine mir zusagende Beschäftigung und macht mir deshalb Vergnügen. ${ }^{16}$

Einen Tag zuvor, am 14. Juli 1877, hatte Leopold Ullstein für 60.000 Mark die Druckerei „Stahl \& Assmann“ in der Zimmerstraße 94 sowie den im gleichen Haus ansässigen Zeitungsverlag des Neuen Berliner Tageblatts, einer redaktionellen Abspaltung von Rudolf Mosses 1871 gegründetem Berliner Tageblatt, erworben. Der Kauf der beiden kurz vor dem Bankrott stehenden Unternehmen „nebst sämtlichem im Geschäftslokal befindlichem Inventar, Geschäftsutensilien, Mobilien, Warenvorräten, überhaupt wie alles steht und liegt“ wie es im Kaufvertrag hieß, schuf die materielle Grundlage für den Ullstein-Verlag, der dann am 1. August 1877 als offene Handelsgesellschaft gegründet wurde. ${ }^{17}$ Die gerichtliche Eintragung erfolgte am 11. September 1877 durch Verkündung des Königlichen Stadtgerichts. Für 60.000 Mark hatte Leopold Ullstein damit ein Unternehmen erworben, dass sechs Jahrzehnte später 60 Mio. Mark wert sein würde.

Wie der gelernte Papierhändler Leopold Ullstein waren auch viele andere Verleger der späteren Massenpresse berufliche Quereinsteiger aus der Publizistik verwandten Branchen, z. B. August Madsack (gelernter Buchdrucker), August Scherl (Verlagsbuchhändler), Rudolf Mosse (Annocenvermittler) oder August Huck (Besitzer einer Schriftgießerei). ${ }^{18}$ Die sich seit 1848 abzeichnende Ex-

16 Faksimile des Briefs von Leopold Ullsteins an seine älteste Tochter, in: Ullstein Verlag, 50 Jahre Ullstein, S. 7.

17 Ullstein Verlag, 50 Jahre Ullstein, S. 7.

18 Vgl. Stöber, Rudolf: Deutsche Pressegeschichte. Von den Anfängen bis zur Gegenwart. Konstanz 2005, S. 256. 
pansion des Berliner Zeitungsmarkts, von der viele von ihnen profitierten, erreichte in den Jahren zwischen der Reichsgründung und dem Ersten Weltkrieg ihre volle Entfaltung: Gab es im Berlin des Jahres 1871 noch zwölf Tageszeitungen, stieg ihre Anzahl bis 1914 auf insgesamt 30 täglich erscheinende Morgenzeitungen, zehn Abendblätter und etwa 50 Vorortzeitungen an. ${ }^{19}$ Vergleichbar waren die Berliner Verhältnisse bestenfalls mit jenen der Zeitungskapitale London.

Das Neue Berliner Tageblatt verkündete in einer Abonnement-Einladung am 1. September 1877 „den Ausbau, die Einheit, die Freiheit und die Macht des deutschen Vaterlandes“ als seine politische Zielsetzung. ${ }^{20}$ Einen Monat später wandelte der neue Besitzer die Zeitung von einem Morgenblatt zu einer Abendzeitung um, bei dieser Gelegenheit änderte Ullstein auch den Namen der Publikation in Deutsche Union. Da das Blatt jedoch nicht florieren wollte, wurde es bald mit der zweiten Publikation des Ullstein-Verlags verschmolzen: der Berliner Zeitung.

Denn etwa zur gleichen Zeit, als Leopold Ullstein die ersten praktischen Erfahrungen im Tageszeitungsgeschäft sammelte, hatte der fortschrittliche Journalist Peter Langemann die Berliner Zeitung gegründet. Als Ullstein von den wirtschaftlichen Problemen der Zeitung, die sich selbst als „unabhängig von allem Cliquen- und Parteiwesen“ bezeichnete, hörte, griff er erneut zu: Am 1. Januar 1878 erwarb der Verleger auch diese Publikation.

Die Zeit war günstig für die Berliner Zeitung, denn der russisch-türkische Krieg hatte das Interesse an neuesten Informationen signifikant erhöht. ${ }^{21}$ Die Berliner Zeitung erhöhte mit Extraausgaben und Sondernummern den Takt der Zeitungsstadt, etwa, als sie am 2. Juni 1878 die Bevölkerung über das Attentat Carl Nobilings auf Kaiser Wilhelm I. unterrichtete. ${ }^{22}$ In den darauffolgenden Tagen präsentierte sie noch ein Abbild des Attentäters samt Vollbart - eine schraffierte Zeichnung auf der ersten Seite, denn Ullstein war von Anfang an der gerechtfertigten Überzeugung, dass die Leser Illustrationen wünschten. ${ }^{23}$

19 Dazu Mendelssohn, Anfänge, S. 55.

20 Vgl. Mendelssohn, Zeitungsstadt, S. 121.

21 Aus: Ein Gott hat uns beschützt. In: Der Spiegel, Nr. 04/1952 vom 23.01.1952, S. 10-17, hier S. 12.

22 Über die Bedeutung von Sonderausgaben siehe Koszyk, 19. Jahrhundert, S. 284.

23 Neben Berlin begann sich zeitgleich in Hamburg die Tendenz zur Pressezeichnung durchzusetzen, vgl. Duttenhöfer, Bettina: Innovationen um 1900. Investigativer und lokaler Journalismus - Frauenjournalismus - Visualisierung. In: Politischer Journalismus, Öffentlichkeiten und Medien im 19. und 20. Jahrhundert. Hrsg. von Clemens Zimmermann. Ostfildern 2006 (Schriften der Siebenpfeiffer-Stiftung 8). S. 139-168, hier S. 149. 
Eine neue Rotationsmaschine ermöglichte zudem, den Redaktionsschluss auf ein Uhr nachts zu verschieben.

Hohe Schnelligkeit, politische Meinungsstärke, Bebilderungen - bei der Berliner Zeitung erprobte Leopold Ullstein einen neuen Typus der Tageszeitung, der bisher in Deutschland unbekannt war. All die redaktionellen Bemühungen zahlten sich schnell aus: Gut ein Jahr, nachdem er die Berliner Zeitung erworben hatte, am 22. Dezember 1878, erreichte sie 19.500 Abonnenten. Am Jahresende 1879 waren es bereits etwa 25.000 Leser, und bald verzeichnete sie schon 40.000 Abonnenten. ${ }^{24}$ Dies war insofern erstaunlich, weil das Berliner Tageblatt und die Berliner Volkszeitung in diesen Tagen ebenfalls genuin das liberale Leser-Spektrum der Reichshauptstadt bedienten. Andererseits konnte die Berliner Zeitung von den Sozialistengesetzen profitieren - da es bis 1884 keine sozialdemokratische Partei in Berlin gab, kam ein nicht unwesentlicher Teil ihrer Leser eben aus diesem Milieu. ${ }^{25}$

Die eigentliche Anziehungskraft der Berliner Zeitung lag nämlich in ihrer entschieden freiheitlichen Haltung. Kaum dass die Zeitung seit einem Dreivierteljahr in Ullstein-Besitz war, druckte sie am 29. September 1878 eine programmatische Erklärung: „Die ,Berliner Zeitung“ will eine konstitutionelle Regierung, keinen Kanzler-Absolutismus. Sie verlangt, dass das deutsche Volk frei sei und nicht wie eine besiegte Nation behandelt werde. “26 Die Folgen dieser Kampfansage ließen nicht lange auf sich warten. Denn mit besonderer Aufmerksamkeit kommentierte die Redaktion diejenigen Debatten im Preußischen Abgeordnetenhaus, in denen sich Bismarcks Kulturkampf widerspiegelte. Die Abgeordneten wiederum „nutzten ihre Immunität, um ungestraft gegen das ,Judenblatt` zu hetzen“. 27

1881 war es der wachsenden Berliner Zeitung zu eng geworden in den Räumen der Zimmerstraße 94. So erwarb Ullstein am 2. Mai das eine Straßenecke weiter gelegene Grundstück Kochstraße Nummer 23 (Grundfläche: 1.038 qm), noch im gleichen Jahr zogen Redaktion und Druckerei um. 1885 begann der Neubau der Hausnummer 23 nach einem Entwurf des Architekten Schwenke. In den kommenden drei Jahrzehnten wuchs der Ullstein-Verlag in solch einem Maße, dass das Unternehmen den ganzen gewaltigen Häuserblock KochstraßeCharlottenstraße-Markgrafenstraße-Besselstraße einnehmen sollte.

Da die Berliner Zeitung nur morgens erschien, standen die beiden für ihre Produktion notwendigen Rotationsmaschinen tagsüber still. Aus kaufmänni-

24 Siehe Ullstein Verlag, 50 Jahre Ullstein, S. 10.

25 Hierzu Koszyk, 19. Jahrhundert, S. 284.

26 Mendelssohn, Zeitungsstadt, S. 126.

27 Vgl. Der Spiegel (04/1952), Gott, S. 12. 
scher Sicht erschien es demnach sinnvoll, auch eine Abendzeitung herzustellen. Da Leopold Ullstein aber den Berliner Markt für noch nicht groß genug für eine weitere Abendzeitung hielt, wollte er die neue Zeitung nicht für die Stadt, sondern das ganze Reich herstellen - am Abend in Berlin gedruckt und für den Versand bereitgestellt, so dass sie am nächsten Morgen in der Provinz zugestellt sein würde. ${ }^{28}$ Die Zeiten für solch einen Plan waren günstig: Erst an wenigen Orten im Reich waren Blätter vorhanden, die es mit der Aktualität der Berliner Publikationen aufnehmen konnten, zudem verbesserte die Eisenbahn stetig ihre Verbindungen und der Postzeitungstarif war mit $20 \%$ des Bezugspreises sehr niedrig. Und schließlich gab es einen großen Bedarf im Reich für Geschichten aus der expandierenden und für deutsche Verhältnisse mondänen Hauptstadt.

Am 1. September 1887 erschien zum ersten Mal die Berliner Abendpost im Reichsgebiet für den Abonnementpreis von einer Mark pro Vierteljahr. In verhältnismäßig kurzer Zeit stieg die Auflage auf 70.000 Exemplare an und übertraf damit jene der Berliner Zeitung deutlich. ${ }^{29}$ Der Erfolg war auch modernem Marketing zu verdanken: In etwa 200 Zeitungen des Deutschen Reichs wurde das Erscheinen der Abendpost großformatig beworben, eine für damalige Verhältnisse unbekannte Dimension. Die offensive Werbung sollte in den kommenden Jahrzehnten zu einem Markenzeichen der Ullstein-Publikationen werden.

Die Herstellung zweier Zeitungen unter einem Dach stellte nicht nur für die Druckerei eine Optimierung ihrer Leistungsfähigkeit dar. Leopold Ullstein beschloss, dass die Verwendung des gesamten redaktionellen Apparates beiden Zeitungen zugute kam: Da die beiden Blätter nicht am gleichen, sondern verschiedenen Orten, nämlich die eine der Hauptstadt, die andere in der Provinz, gelesen wurden, konnte ein Großteil des redaktionellen Materials zweimal verwendet werden. Dieser doppelte Rationalisierungsvorgang, bei der Herstellung und dem Inhalt, sorgte für deutliche Umsatzsprünge beim Ullstein-Verlag. ${ }^{30}$

Auch bei den Anzeigen beschloss man eine Kooperation der beiden Erfolgsblätter: Als am 25. April 1889 zum ersten Mal die Abonnenten-Zahlen der Berliner Zeitung und der Berliner Abendpost eine Gesamtziffer von 100.000 Exemplaren erreichten, kündigte der Verlag an, den zu diesem Zeitpunkt bereits stattli-

28 Dazu schrieb Leopold Ullstein an seinen Sohn Louis am 19.09.1885: „Deine Ansichten über die Berliner Zeitung sind ganz richtig, nur muss man das Sichere nicht gegen das Unsichere gefährden. Dieses ist die Berliner Zeitung als Lokalblatt für Berlin und das will ich nicht für das Unsichere (auswärts) vernachlässigen. Überhaupt ist eine Zeitung für Auswärts und für Berlin nicht zu vereinigen. Deshalb projektiere ich immer noch die Abendzeitung für Außerhalb.“ Aus: Ullstein Verlag, 50 Jahre Ullstein, S. $28 \mathrm{f}$.

29 Dazu Mendelssohn, Zeitungsstadt, S. 160.

30 Mendelssohn, Zeitungsstadt, S. 162. 
chen Kleinanzeigenteil der Berliner Zeitung auch in der Abendpost zu drucken $^{31}$ - und konnte seinen Anzeigenkunden auf diese Weise eine sechsstellige Leserzahl garantieren.

Am 18. Januar 1889 traten die beiden ältesten Söhne, Hans und Louis, als Teilhaber in den Ullstein-Verlag ein. Ihrem Beispiel folgten in den kommenden Jahren auch die anderen drei Brüder Franz (Mitinhaber seit 1897), Rudolf (seit 1901 Teilhaber) und Hermann (seit 1902 Teilhaber). Jeder der fünf Ullstein-Söhne, also die zweite Verleger-Generation, übernahm einen bestimmten Aufgabenbereich, der seinen Neigungen und Interessen entsprach. Dieses Zusammenspiel der fünf sehr verschiedenen Charaktere war maßgeblich verantwortlich für den Aufstieg des Ullstein-Verlags zu einem der führenden publizistischen Häuser Europas. Die zunehmende Technisierung des Herstellungsprozesses und die Ausrichtung der Zeitungen auf den Geschmack einer immer größer werdenden Masse erforderten „ohnehin den Verleger-Typus des kalkulierenden Managers". 32

Einen Überblick über die Fähigkeiten und die daraus resultierenden Aufgabenbereiche der Brüder gab der jüngste Verlegersohn, Hermann Ullstein, 1943 während der Emigration in den USA. ${ }^{33}$ Hans hatte Rechtswissenschaften studiert und war wie sein Vater politisch engagiert: Er saß für die Fortschrittliche Volkspartei zwischen 1904 und 1911 sowie von 1912 bis 1919 in der Stadtverordnetenversammlung des Berliner Rathauses. Als Chef der Berliner Zeitung und später der B.Z. am Mittag verfasste er regelmäßig Leitartikel - und als Jurist hatte Hans die Blätter hin und wieder persönlich vor Gericht vertreten. Nach dem Tode seines Vaters Leopold wurde Hans zunächst der Seniorchef des Hauses. Mit der Umwandlung des Unternehmens in eine Aktiengesellschaft übernahm er 1921 den Vorsitz des Aufsichtsrats, zwischen 1930 und 1933 hatte er den Ehrenvorsitz dieses Gremiums inne. ${ }^{34}$

Louis wiederum hatte bei den Dragonern gedient und war gelernter Kaufmann, vor allem die Verlagsgeschäftsführung wurde sein Metier. Er gehörte dem Aufsichtsrat der AG zwischen 1921 und 1930 als stellvertretender Vorsitzender an. Franz Ullstein wurde in Rechtswissenschaften promoviert, 1894 trat er in den väterlichen Verlag ein. Ab 1921 wurde Franz der Vorstandsvorsitzende der Ullstein AG, diesen Posten hatte er bis 1930 inne. Dr. Franz Ullstein war der

31 Hierzu: Ullstein Verlag, 50 Jahre Ullstein, S. 33. Der Ullstein-Verlag bediente sich bei der redaktionellen Befüllung seiner verschiedenen Publikationen eines Redaktionspools, der im Jahr 1900 lediglich 37 Redakteure umfasste. Vgl. Faulstich, Werner: Medienwandel im Industrie- und Massenzeitalter (1830-1900). Göttingen 2004 (Geschichte der Medien 5), S. 32.

32 Siehe Koszyk, 19. Jahrhundert, S. 228.

$33 \mathrm{Vgl}$. Ullstein, Hermann: Haus Ullstein, S. $71 \mathrm{f}$.

34 Siehe Laabs, Verlegerfamilie, S. 576. 
Leiter der Tageszeitungssparte, verlegerisch hatte er aber in allen Bereichen das letzte Wort. Nach einem kurzen Zwischenspiel als Chef des Aufsichtsrats im Jahr 1933 musste er ein Jahr später - wie alle seine noch lebenden Brüder - das mittlerweile zwangsverkaufte Unternehmen verlassen.

Rudolf trat 1901 in das Familienunternehmen ein: Im Flemming-Haus in Glogau, einem der größten schlesischen Verlagshäuser, war er zu einem Druckereifachmann ausgebildet worden - so ernannte ihn Leopold Ullstein zum technischen Direktor. Zwischen 1921 und 1930 war er Mitglied des Aufsichtsrats der Ullstein AG, von 1930 bis 1933 hatte er den Posten des stellvertretenden Vorsitzenden inne.

Hermann Ullstein schließlich, der jüngste Bruder, wollte eigentlich ganz andere berufliche Wege gehen, so absolvierte er seine kaufmännische Lehrzeit bei einem Getreidekonzern. Doch ab 1902 arbeitete er in der Zeitschriftenabteilung des Verlags, von 1924 bis 1927 amtierte Hermann als stellvertretender Vorstandsvorsitzender der Ullstein AG, zwischen 1927 und 1933 gehörte er dem Aufsichtsrat an. Der Familienverband befand, der „Künstler“ unter den fünf Söhnen habe ein Talent für Reklame - oder für „Propaganda“, wie man damals sagte.

Das Zusammenspiel dieser fünf Charaktere lief wahrlich nicht reibungslos in den kommenden Jahren. Und dennoch: Unter der Leitung der fünf UllsteinBrüder erlebte das Unternehmen einen nie gekannten Aufschwung. ${ }^{35}$ Gegen Ende der 1880er Jahre traten alle zehn Kinder Leopold Ullsteins zum Protestantismus über, die Enkelgeneration und deren Nachkommen wuchsen im evangelischen Glauben auf. ${ }^{36}$ Alleinige Ausnahme war Heinz Ullstein (1893-1973), Louis' ältester Sohn: Er hatte als einziger Ullstein während des Zweiten Weltkriegs in Berlin ausgeharrt und war in dieser Phase der permanenten Lebensgefahr zum Katholizismus übergetreten. ${ }^{37}$ Es sollte nicht der einzige Sonderweg Heinz Ullsteins bleiben.

Der nächste große Coup gelang auf Umwegen zu den Ullsteins. Im Jahr 1891 hatte der Verlag in der Markgrafenstraße unter Louis' Leitung eine Akzidenzdruckerei eröffnet, die neben dem eigenen Bedarf auch fremde Druckaufträge ausführte. Einer jener Fremdaufträge war die seit einem Jahr erscheinende Berliner Illustrirte Zeitung. ${ }^{38}$ Ihr Besitzer, ein schlesischer Kaufmann namens Hep-

35 Vgl. hierzu Der Spiegel (04/1952), Gott, S. 14.

36 Siehe Münzel, Martin: Die jüdischen Mitglieder der deutschen Wirtschaftselite 1927-1955. Verdrängung - Emigration - Rückkehr. Dissertation. Paderborn 2006, S. 89.

37 Vgl. hierzu Frederick Ullstein, Hundert Jahre, S. 38.

38 Siehe Mendelssohn, Zeitungsstadt, S. $164 \mathrm{f}$. 
ner, hatte zwar den Bedarf nach einem bebilderten Blatt erkannt, besaß gleichwohl keine journalistische Erfahrung.

Hepners Geschäftspartner, der mit Ullstein befreundete Otto Eysler, trat nun mit dem Druckauftrag an die neue Akzidenzdruckerei heran - und weckte das Interesse Ullsteins. Leopold erwarb die Illustrirte und umging das umständliche, auf langwierige Engagements ausgerichtete Abonnementsystem, indem er den Verkauf frei Haus für zehn Pfennige pro Nummer (der Straßenverkauf war noch polizeilich stark eingeschränk $\mathrm{t}^{39}$ ) ermöglichte. Damit einher ging eine so interessante Text- und vor allem Fotogestaltung - die technischen Voraussetzungen dafür waren eben erst erfunden worden -, dass der Leser tatsächlich freiwillig die nächste Ausgabe kaufte..$^{40}$

Die Berliner Illustrirte galt als Ullsteins größter Erfolg. ${ }^{41}$ Zunächst verzeichnete sie 60.000 Abonnenten, zur Jahrhundertwende waren es bereits 100.000; als 1904 dann der Straßenvertrieb von Zeitungen freigegeben wurde, als also der Kiosk sowie der mobile Zeitungsverkäufer möglich wurden, war der Weg zum Massenblatt und damit ein Auflageanstieg möglich, der 1931 schließlich zwei Mio. Exemplare erreichen sollte.

\subsection{Der erste Berliner Zeitungskrieg}

Bereits 1883 hatte Ullsteins schärfster Konkurrent, August Scherl, den Lokal-Anzeiger gegründet, den er durch ein Heer von eigenen Verteilern frei ins Haus

39 Hierzu Stöber, Pressegeschichte, S. 260, der darauf hinweist, dass zwar 1848 der Straßenverkauf in Berlin gegen „fliegende Händler“ durch mehrere Prozesse untersagt worden war; gleichwohl war der Straßenverkauf per se laut des Preußischen Pressegesetzes von 1851, § 10, eben nicht verboten, sondern von der Erlaubnis der Ortspolizeibehörden abhängig.

40 Friedrich Luft schrieb über den Erfolg der Berliner Illustrirten: „Es gab schon in den ersten,

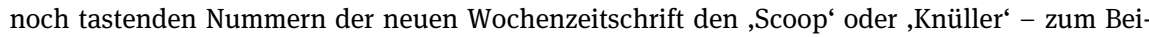
spiel wenn man den Papst, Leo XIII., beim Besprechen der ersten Phonographenrolle abbildete. Man erkennt schon den Reiz, den die Abbildungen von Zeitberühmtheiten in ihrer intimen Umgebung auslöst. Wenn der Erfolgsdramatiker Hermann Sudermann oder wenn der alte Rudolf Virchow sozusagen ,im Gehäuse‘ fotografiert werden, ist mit solcher Nähe und Intimität des Bildes etwas Sensationelles, zuvor Unmögliches geleistet. In Zeichnung und Xylographie waren solche Annäherungen, so glaubhafte Dokumentationen berühmter oder profaner Menschlichkeiten nicht zu schaffen. Jetzt wurde das möglich.“ Aus: Luft, Friedrich: Berliner Illustrirte. In: Hundert Jahre Ullstein 1877-1977. Band 2. Hrsg. von Joachim W. Freyburg u. Hans Wallenberg. Frankfurt a. M./Berlin/Wien 1977. S. 87-118, hier S. 91.

41 Vor dem Erfolg der BIZ gab es zahlreiche gescheiterte Illustrierten-Gründungen, etwa die Deutsche Illustrirte Zeitung (1884-1887) oder Der Reporter (1895-1902), vgl. hierzu: Weise, Bernd: Pressefotografie II. Fortschritte der Fotografie- und Drucktechnik und Veränderungen des Pressemarktes im Deutschen Kaiserreich. In: Fotogeschichte 33 (1989). S. 27-62, hier S. 49. 
bringen ließ. Doch nicht im Vertriebswesen stellte der Lokal-Anzeiger eine Novität dar: Statt langer erklärender Artikel setzte Scherl hier auf telegrafische Nachrichten aus aller Welt, druckte Kriminetten und Boulevardnachrichten und baute den Lokalteil kräftig aus, „,volkstümlich“ hieß das damals. Der Lokal-Anzeiger machte Scherls Konkurrenten zu schaffen. Die Zeitung gehörte zu den erfolgreichsten des sich durchsetzenden Generalanzeiger-Typus, der sich neben dem breiten Adressaten-Spektrum durch eine Mischfinanzierung von Abonnementund Anzeigenerlösen auszeichnete. ${ }^{42}$ Er erreichte noch vor dem Ersten Weltkrieg ein Verhältnis von Anzeigen- und Verkaufserlös von $70 \mathrm{zu} 30 .{ }^{43}$

Am 20. September 1898 erschien Ullsteins Replik - nämlich die erste Ausgabe der Berliner Morgenpost, die für zehn Pfennige pro Woche frei Haus geliefert wurde. Die neue Zeitung präsentierte sich mit ihrer direkten Sprache bewusst als Gegenprodukt zur vermeintlich farblosen Generalanzeiger-Presse. ${ }^{44}$ Mit der Morgenpost begann der Zeitungskrieg Ullstein gegen Scherl, der Berlin bis in das neue Jahrhundert hinein bewegte. Freilich wurde die Auseinandersetzung vor allem durch Plakate geführt: Bereits nach zwei Wochen hatte die Berliner Morgenpost eine Auflage von 40.000 Exemplaren, Tendenz steigend. Mit werbewirksamen Anschlägen des populären Zeichners Edmund Edel, die an alle Litfaßsäulen geklebt wurden, hatte Ullstein sein neuestes Produkt in der Stadt bekannt gemacht. Scherl nahm die Herausforderung an, Woche für Woche erschienen nun die - deutlich langsamer ansteigenden - Verkaufszahlen des Lokal-Anzeigers auf den Säulen. Ullstein konterte und kündigte mit ebenso großen Plakaten die deutlich schneller ansteigende Zahl der Morgenpost-Abonnenten an. ${ }^{45}$

Bereits nach sieben Monaten hatte die Morgenpost eine Auflage von knapp 100.000 Exemplaren, nach neun Monaten holte sie mit 120.000 Abonnenten den Lokal-Anzeiger ein. Fast ein Jahr nach seiner Gründung, am 17. September

42 Als entscheidendes Abgrenzungsmerkmal des Generalanzeiger-Typus' zu den bisherigen Publikationen sieht Requate deutlich weniger die Anzeigenfinanzierung als vielmehr die Konzeption für neue Publikumsschichten, siehe Requate, Jörg: Kommerzialisierung der Presse im frühen 20. Jahrhundert. Konsumierendes und fragmentiertes Publikum. In: Politischer Journalismus, Öffentlichkeiten und Medien im 19. und 20. Jahrhundert. Hrsg. von Clemens Zimmermann. Ostfildern 2006 (Schriften der Siebenpfeiffer-Stiftung 8). S. 121-138, hier S. 125.

43 Vgl. Stöber, Pressegeschichte, S. 258.

44 Siehe Requate, Kommerzialisierung, S. 133 f. Requate betont zudem, dass die Entstehung der Boulevardpresse eben nicht eine weitere Depolitisierung bedeutete: „Parteinahme und kommerzieller Erfolg, so konnte man als Lehre aus der Auflagenentwicklung der ,Morgenpost“ ziehen, schlossen sich keineswegs aus.“

45 Vgl. Wagner, Rainer: Berliner Morgenpost. In: Hundert Jahre Ullstein 1877-1977. Band 2. Hrsg. von Joachim W. Freyburg u. Hans Wallenberg. Frankfurt a. M./Berlin/Wien 1977. S. 9-46, hier S. 15. 
1899, verzeichnete das Ullstein-Blatt etwa 160.000 Bezieher. ${ }^{46}$ Ullstein hatte den ersten Zeitungskrieg der deutschen Geschichte für sich entschieden. Die marktbeherrschende Stellung des Lokal-Anzeigers und der Berliner Morgenpost führte dazu, dass mehr als die Hälfte aller Berliner regelmäßig eine der beiden Zeitungen las. ${ }^{47}$

Nach diesem Sieg entsandte Scherl, der genau wusste, welch immense Ausgaben seine Konkurrenten durch das neue Prestigeobjekt hatten, einen Unterhändler zu den Ullsteins. Sein Angebot: Scherl wollte einen Anteil an der Morgenpost erwerben. Seine Bedingungen: Der teure Reklamekrieg an den Litfaßsäulen soll beendet werden und der Ullstein-Verlag dürfe keine Auflagenzahlen mehr veröffentlichen. Die Ullsteins erkannten in dem Vorschlag auch die Möglichkeit zur eigenen finanziellen Konsolidierung, denn nun konnten sie ungestört von der Konkurrenz den Preis der Berliner Morgenpost von zehn auf 15 Pfennige pro Woche erhöhen ${ }^{48}$ - damit lagen sie noch immer deutlich unter dem Preis des Lokal-Anzeigers, der sich auf eine Mark pro Monat belief. Das Preisdumping hatte für die Ullsteins hier noch einmal ein gutes Ende genommen.

Jetzt aber einigte man sich schnell: Die Morgenpost wurde aus dem Ullstein-Verlag ausgegliedert und in eine Gesellschaft mit beschränkter Haftung umgestaltet. Das Stammkapital dieser Berliner Morgenpost $\mathrm{GmbH}$ betrug 1,25 Mio. Mark, es wurde von den Firmen Scherl und Ullstein zu gleichen Teilen, also je 625.000 Mark, gestellt. Die Partnerschaft Scherl-Ullstein, geschlossen im April 1900, wurde bereits zwei Jahre später wieder nahezu gelöst, denn dann konnte Ullstein die Hälfte der Scherlschen Einlage zu ihrem Nennwert zurückkaufen. Komplett beendet wurde die Kooperation 1909, als Ullstein wieder alle Anteile an der Berliner Morgenpost besaß. Durch die Finanzspritze hatte sich

46 Auf der Seite der Berliner Morgenpost erschien an jenem 17.09.1899 ein Faksimile der handschriftlichen Beglaubigung des gerichtlichen Bücherrevisors Klebba mit folgenden Wortlaut: „Auf Grund der mir vorgelegten und von mir geprüften Bücher und Belege bescheinige ich hiermit, dass die Berliner Morgenpost jute 154.349 Abonnenten hat. Nicht inbegriffen sind die durch fremde Spediteure und im Einzelhandel abgegebenen, sowie die Frei- und Belegexemplare.“, siehe Wagner, Morgenpost, S. 15.

47 Siehe hierzu Fritzsche, Peter: Als Berlin zur Weltstadt wurde. Presse, Leser und die Inszenierung des Lebens. Berlin 2008, S. 115.

48 Louis Ullstein soll, als er das Angebot Scherls mit seinen Brüdern besprach, ausgerufen haben: „Das ist unsere Rettung!“, nach Mendelssohn, Zeitungsstadt, S. 202. Hermann Ullstein: „Die ,Morgenpost‘ verschlingt Unsummen. Wenn das so weitergeht, haben wir bald alles verloren. Was auch geschieht, wir müssen die Preise erhöhen. 10 Pfennig für eine wöchentliche Lieferung der ,Morgenpost“ sind zu wenig. Wenn wir einen Vertrag mit Scherl abschließen, können wir nicht nur unsere Liquidität sicherstellen, sondern ohne Gefahr den Preis anheben.“ Nach Ullstein, Haus Ullstein, S. $86 \mathrm{f}$. 
die Zeitung finanziell konsolidieren können, Scherl hatte lediglich an ihrem weiteren Aufstieg mitverdient. Unabhängig davon blieb ein Teil des Abkommens zwischen Scherl und Ullstein aus dem April 1900 unverändert bestehen: Wenn einer der beiden ein neues Projekt startete, verpflichtete sich der andere, nichts Ähnliches herauszubringen. ${ }^{49}$ Jener „Freundschafts- und Konkurrenzausschluss-Vertrag“ wurde erst 1914 wieder gelöst. Von Ullsteinscher Seite hatten Leopold Ullsteins fünf Söhne diese Übereinkunft mit August Scherl getroffen, denn ihr Vater hatte den Anbruch des neuen Jahrhunderts nicht mehr erlebt.

In der Nacht zu Sonntag, dem 4. Dezember 1899, verstarb Leopold Ullstein im Alter von 73 Jahren. Unter großer öffentlicher Anteilnahme fand die Beisetzung am 6. Dezember auf dem jüdischen Friedhof an der Schönhauser Allee statt. Leopold Ullstein starb als einer der drei großen Zeitungsverleger nicht nur Berlins, sondern Deutschlands. Ullstein, Mosse und Scherl, die ersten zwei mit liberaler, letztgenannter mit konservativer Ausrichtung, bestimmten maßgeblich die publizistische Landschaft des Kaiserreichs. Anders aber als Mosse hatte sich Ullstein erfolgreich auf die Verbreitung von modernen Massenblättern verlegt, die den Spagat zwischen Partei- und Geschäftspresse meisterten.

Leopold Ullsteins Verlag war unter den Großen der innovativste, so quecksilbrig wie Berlin selbst, eine Grundstimmung, die Thomas Mann einmal als „preußisch-amerikanisch“ bezeichnet hatte. ${ }^{50}$ Mit dem Beginn des neuen Jahrhunderts war jedoch auch das Ende der Gründerzeit erreicht: Für die Publizistik bedeutete dies, dass natürlich auch in den kommenden drei Jahrzehnten zahlreiche Zeitungen in Berlin gegründet wurden - vor allem wieder zwischen 1924 und 1929. ${ }^{51}$ Aber diese hatten es jetzt bedeutend schwerer, sich durchzusetzen. Die Gründerzeit, in der aus einer zündenden Idee ein Imperium entstehen konnte, war vorbei.

Ein ganz großer Wurf aber gelang noch im Berliner Zeitungsviertel, jetzt, zwischen Jahrhundertwende und Erstem Weltkrieg.

49 Vgl. hierzu Der Spiegel (04/1952), Gott, S. 12.

50 Siehe Stölzl, Ullstein-Geist, S. 10.

51 Bis zum Ersten Weltkrieg stieg das Interesse an Zeitungen deutlich an: Zwischen 1878 und 1913 wuchs die Berliner Bevölkerung um ca. 75 \%, die Gesamtauflage der hier erscheinenden Zeitungen aber sogar um nahezu 679 \%. Nach Gossel, Daniel: Medien und Politik in Deutschland und den USA. Kontrolle, Konflikt und Kooperation vom 18. bis zum frühen 20. Jahrhundert. Stuttgart 2010 (Transatlantische Historische Studien 35), S. 113. 


\subsection{Konsolidierung der Marktposition und Erweiterung des Portfolios}

Die Berliner Zeitung drohte zum Stiefkind des Ullstein-Verlags zu werden. ${ }^{52}$ Zwar erschien sie mittlerweile zweimal täglich und profitierte vom Speditionsvertrieb der Berliner Morgenpost. Aber in ihrer Aufmachung und inhaltlichen Darstellung wirkte sie nach den vergangenen Eruptionen auf dem Berliner Zeitungsmarkt bereits veraltet. Als 1904 der Straßenverkauf freigegeben wurde ${ }^{53}$, war es Louis Ullstein, der die Chance einer Mittagszeitung sah, die mit der Abendausgabe der Berliner Zeitung verschmolzen werden würde. ${ }^{54}$ Das neue Produkt sollte zunächst Berliner Zeitung am Mittag heißen, bis Hans Ullstein die Abkürzung B.Z. am Mittag vorschlug. Ihre erste Nummer erschien am 22. Oktober 1904 - es war das erste ganz auf den Straßenverkauf eingestellte Blatt Deutschlands seit 1848. ${ }^{55}$ Nachdem sein Gelingen gesichert war, stellte man das Erscheinen der Berliner Zeitung als Abonnement-Blatt zum April 1905 völlig ein.

Auch bei der B.Z. am Mittag erwiesen sich die Ullsteins als Marketing-Experten: Zeitungsverkäufer mit besonderen Uniformen, Werbung an Bauzäunen und Häuserwänden steigerten die Verkaufsziffern schnell. ${ }^{56}$ Überhaupt begann in diesen Jahren die Werbung ihren Siegeszug in Deutschland - und traf auf vielfältige Ablehnung, wobei sich hier anti-amerikanische Ressentiments mit antisemitischen Stereotypen verbanden.

Je mehr sich Reklame in der Presse oder bei Warenhäusern mit jüdischen Erfolgsunternehmen (Ullstein, Tietz, Wertheim) verbinden ließ, desto stärker wuchs die Agitation gegen sie, etwa durch Diffamierungskampagnen. ${ }^{57}$ Die Reklame wurde „zum Synonym der als bedrohlich empfundenen Moderne“. 58

Dem Mitbewerber Scherl waren durch den „Freundschafts- und Konkurrenzausschluss-Vertrag“ die Hände gebunden, eine weitere Straßenzeitung war

52 Siehe hierzu: Ullstein Verlag, 50 Jahre Ullstein, S. 48.

53 Stöber, Pressegeschichte, S. 260, weist darauf hin, dass die B.Z. am Mittag nicht die erste Straßenverkaufzeitung - mithin Boulevard-Zeitung - in Deutschland war, da es bereits 1848 die kurzlebige Berliner Straßenzeitung gegeben hatte.

54 Vgl. auch Wagner, Erich u. Peter Fritz: BZ am Mittag und BZ. In: Hundert Jahre Ullstein 1877-1977. Band 2. Hrsg. von Joachim W. Freyburg u. Hans Wallenberg. Frankfurt a. M./Berlin/ Wien 1977. S. 47-86, hier S. 50.

55 Obwohl im Straßenverkauf erhältlich, wies sich die B.Z. am Mittag als seriös aus, im Vergleich vor allem mit den zu dieser Zeit bereits in den USA und Großbritannien gängigen Sensationsblättern der „Yellow Press“. Hierzu: Wilke, Jürgen: Grundzüge der Medien- und Kommunikationsgeschichte. Von den Anfängen bis ins 20. Jahrhundert. Köln/Weimar/Wien 2000, S. 270 .

56 Siehe hierzu Der Spiegel (04/1952), Gott, S. 12.

57 Vgl. Gossel, Medien und Politik, S. 115. 
also von ihm nicht zu erwarten. Bekannt wurde die B.Z. am Mittag als „schnellste Zeitung der Welt“: Ihr rastloser Rekord, Aktualität binnen dreißig Minuten von der Ankunft der Nachricht bis zum Vertrieb auf die Straßen der Reichshauptstadt zu bringen, wurde auf Plakaten propagiert - und kündete vom Rausch der Geschwindigkeit, dem die Gesellschaft im unruhigen ersten Jahrzehnt des 20. Jahrhunderts verfallen war. ${ }^{59}$

Rudolf Ullstein erfand 1911 mit dem „B. Z.-Preis der Lüfte“ den ersten Rundflug über Deutschland. Aktionen wie diese machten Ullstein im ganzen Reich als Marke bekannt, verschlangen aber viel Geld. Geld, das zumeist von den weniger auffälligen Abteilungen des Verlags erwirtschaftet wurde. ${ }^{60}$

Im Jahr 1905 erwarben die Ullsteins die Zeitschrift Dies Blatt der Hausfrau. Neben ihr entwickelte man Die Praktische Berlinerin und beide wurden schließlich im Verlag der Ullstein-Schnittmuster zusammengefasst. ${ }^{61}$ Mit Ullsteins Blatt der Hausfrau (wie die erworbene Zeitschrift inzwischen hieß) führte der Verlag die Schnittmuster ein. Konzeptionell kombinierte dieser neue Typus der Frauenzeitschrift die bisherige Mode-Tradition mit praktischer Ratgeber-Funktionalität. $^{62}$

Fünf Jahre später erwarb der Verlag noch die Modewelt und die zu ihr gehörige Illustrierte Frauen-Zeitung von der Verlagsanstalt „F. A. Bruckmann“. Die Illustrierte Frauen-Zeitung wurde von den Ullsteins zur Neuerscheinung „Dame“ umfunktioniert, die den Vergleich mit dem britischen Tatler nicht zu scheuen brauchte.

In die Zeit der Entwicklung der Frauenzeitschriften fällt auch der Ausbau des Ullstein-Buchverlags. Bereits seit den frühen Tagen der Berliner Zeitung gab es mit Prämienverlegern Lieferverträge über Bücher zu Vorzugspreisen. Zudem waren schon in der Berliner Morgenpost erschienene Serien zu Büchern zusammengefasst worden, 1903 wurde ein eigener Ullstein-Buchverlag gegründet. Unter der Führung des Verlagsleiters Emil Herz entwickelte man ab 1904 den ers-

58 Siehe Lamberty, Christiane: Reklame in Deutschland 1890-1914. Wahrnehmung, Professionalisierung und Kritik der Wirtschaftswerbung. Berlin 2000 (Beiträge zur Verhaltensforschung 38), S. 430.

59 Ausführlich bei Blom, Philipp: Der taumelnde Kontinent. Europa 1900-1914. München 2009. Geschwindigkeitsbedingt hatte die B.Z. am Mittag auch ihren ersten großen journalistischen Coup landen können, als sie am 28.10.1908 das Daily Telegraph-Interview des Kaisers veröffentlichte und damit die Presseabteilung des Auswärtigen Amtes vor eine schwierige Entscheidung stellte. Vgl. hierzu: Winzen, Peter: Das Kaiserreich am Abgrund. Die Daily Telegraph-Affäre und das Hale-Interview von 1908. Darstellung und Dokumentation. Stuttgart 2002 (Historische Mitteilungen 43).

60 Siehe hierzu Der Spiegel (04/1952), Gott, S. 15.

61 Vgl. hierzu Ullstein Verlag, 50 Jahre Ullstein, S. $59 \mathrm{f}$.

62 Siehe Duttenhöder, Innovationen um 1900, S. $151 \mathrm{f}$. 
ten großen Erfolg, das sechsbändige Werk Ullsteins Weltgeschichte - renommierte Wissenschaftler wie Ernst Haeckel, Karl Lamprecht, Wilhelm Oncken oder Emil Brandenburg hatten hierfür Beiträge verfasst. Der nächste Coup war die 1904 gestartete Reihe Musik für alle, sie brachte größtenteils Noten für Klaviermusik heraus, „zweihändig mit überlegtem Text, leicht spiel- und singbar“. ${ }^{63}$ Monatlich erschien ein Heft à 32 Seiten für 50 Pfennig. Bis 1927 kletterte die Auflage der Musik für alle auf 420.000 Exemplare. Zahlreiche weitere populärwissenschaftliche Reihen rundeten das Angebot ab.

Doch auch in der schöngeistigen Literatur wurde Ullstein aktiv: Franz Ullstein beschloss 1909 in Deutschland Billigbücher einzuführen. Zu diesem Zeitpunkt, seit Oktober 1908, hatte Samuel Fischer mit seiner Bibliothek zeitgenössischer Romane zu je einer Mark pro Buch bereits gezeigt, dass der Markt für anspruchsvolle, aber günstige Literatur durchaus vorhanden war. ${ }^{64}$

Die Berliner brachten 1910 eine eigene Romanbibliothek für eine Mark pro Buch auf den Weg, aufgrund der hohen Nachfrage musste deren Auflage relativ schnell auf 100.000 Exemplare erhöht werden. Das erste Ullstein-Buch, Clara Viebigs Dilettanten des Lebens, erreichte eine Gesamtauflage von 283.970 Exemplaren. ${ }^{65}$ Doch das war erst der Beginn einer publizistischen Erfolgsgeschichte: Die Eine-Mark-Bücher von Ullstein-Autoren wie Vicky Baum erreichten Millionen-Auflagen. ${ }^{66}$ Die in ihrem literarischen Anspruch deutlich schwankenden Bände riefen nicht nur Lob hervor; nicht zuletzt mit Blick auf die vom UllsteinBuchverlag herausgegebenen Bücher warnte Samuel Fischer 1911 anlässlich des 25-jährigen Jubiläums seines Verlags vor der Massenproduktion per se und der

63 Zitiert nach Schwab-Felisch, Hans: Bücher bei Ullstein. In: Hundert Jahre Ullstein 18771977. Band 1. Hrsg. von Joachim W. Freyburg u. Hans Wallenberg. Frankfurt a. M./Berlin/ Wien 1977. S. 179-216, hier S. 181.

64 Vgl. Steindl, Gertraude: Samuel Fischer (1859-1934). In: Deutsche Presseverleger des 18. bis 20. Jahrhunderts. Hrsg. von Heinz-Dietrich Fischer. Pullach 1975 (Publizistisch-Historische Beiträge 4). S. 274-284, hier S. 280.

65 Tabellarische Aufstellung aller Verkaufserfolge der Ullsteinbücher in: Ullstein Verlag, 50 Jahre Ullstein, S. 90. Im Gegensatz zu Samuel Fischer konnten die Ullsteins auf keine verlagseigene Liste von Erfolgsautoren zurückgreifen. Zu den Problemen am Anfang erinnerte sich Emil Herz: „Wir hatten beträchtliche Schwierigkeiten, sie [die Autoren, d. Verf.] für die neue Sammlung zu gewinnen, denn sie waren ihrem bisherigen Verleger auf Grund der Verträge mit der gesamten Produktion verpflichtet, und dieser bestand unnachgiebig auf seinem Alleinrecht [...] Alles, was wir erreichen konnten, war die Genehmigung einiger älterer, zum Teil vergriffener Werke.“, aus: Schwab-Felisch, Bücher bei Ullstein, S. 193.

66 Siehe hierzu Erlebnisbericht der Autorin: Baum, Vicky: Meine Zeit mit den Ullsteins. In: Hundert Jahre Ullstein 1877-1977. Band 1. Hrsg. von Joachim W. Freyburg u. Hans Wallenberg. Frankfurt a. M./Berlin/Wien 1977. S. 295-324. 
damit einhergehenden Gefahr einer Verelendung des billigen Buches, die Fischer selbst als „Hintertreppenromantik“ bezeichnete. ${ }^{67}$

Die Ullsteins sahen zudem einen wachsenden Bedarf an aktuellen Fachzeitschriften, so gründeten sie 1910 die Bauwelt, die neben dem Baugeschäft auch den Grundstücks- und Hypothekenmarkt bediente. ${ }^{68} \mathrm{Zu}$ der in Branchenkreisen überaus erfolgreichen Bauwelt gesellte sich 1912 die Holzwelt, zudem übernahmen die Ullsteins die im 30. Jahrgang erscheinende Zeitschrift für Transport und Straßenbau aus dem Julius-Engelmann-Verlag. ${ }^{69}$

1904, dem Jahr, in dem also „die schnellste Zeitung der Welt“ erfunden wurde, feierte Berlins ältestes Tageblatt, die Vossische Zeitung, bereits ihren 200. Geburtstag. ${ }^{70}$ Den Titel als meinungsführende Zeitung der Hauptstadt hatte die Voss zu dieser Zeit schon an die erfolgreicheren Blätter von Ullstein, Scherl und Mosse verloren. Seit 1910 war man aufgrund der desolaten finanziellen Verhältnisse der Zeitung auf der Suche nach einem neuen Eigentümer. ${ }^{71} 1913$ wird die Vossische Zeitung den Ullsteins offiziell angeboten. Kaufpreis: 8 Mio. Mark für die renommierteste Zeitung des Reichs - deren Abonnentenzahl sich zu diesem Zeitpunkt freilich bei ausbaufähigen 25.000 Beziehern eingependelt hatte. ${ }^{72}$ Die Ullsteins waren sich des Risikos des Erwerbs bewusst, denn das Blatt bedurfte einer Generalüberholung. Im Spätherbst 1913 erwarben die Ullsteins die

67 Vgl. Koszyk, Kurt: Der jüdische Beitrag zum deutschen Presse- und Verlagswesen. In: Jüdische Unternehmer in Deutschland im 19. und 20. Jahrhundert. Hrsg. von Werner Mosse u. Hans Pohl. Stuttgart 1992 (Zeitschrift für Unternehmensgeschichte, Beiheft 64). S. 196-218, hier S. 211.

68 Ausführlich dazu: Jaeger, Roland: Die Produktfamilie Bauwelt: Architektur im Programm des Ullstein-Konzerns. In: „Der ganze Verlag ist einfach eine Bonbonniere“. Ullstein in der ersten Hälfte des 20. Jahrhunderts. Hrsg. von David Oels u. Ute Schneider. Berlin/München/ Boston 2015 (Archiv für Geschichte des Buchwesens 10). S. 109-136.

69 Dazu: Ullstein Verlag, 50 Jahre Ullstein, S. $76 \mathrm{f}$.

70 Dagegen wandte Mendelssohn, Zeitungsstadt, S. 42 ff., unter Berufung auf Ernst Consentius' Schrift Die älteste Berliner Zeitung (1928), ein, dass die Vossische eben nicht, wie in ihrem Titelkopf angegeben, 1704 begründet wurde; nicht jene in diesem Jahr von Johann Michael Rüdiger gegründete Zeitung sei die Vorgängerin der Voss, sondern die von seinem Sohn Johann Andreas Rüdiger seit 1721 herausgegebene Königlich prvilegirte Berlinische Zeitung. Diese wiederum gehe zurück auf eine Zeitungsgründung von Johann Lorentz, die schließlich ihren Vorläufer in der ersten Berliner Zeitung, der sogenannten Frischmannschen Zeitung, von 1617 hatte. Mendelssohn: „Die ,Vossische Zeitung“ war die langlebigste Berliner Zeitung. Sie war nahezu hundert Jahre älter, als sie es wusste, und, genau genommen, überhaupt die erste Zeitung Berlins.“

71 Mendelssohn, Zeitungsstadt, S. 255.

72 Bei Bender, Klaus: Vossische Zeitung, Berlin (1617-1934). In: Deutsche Zeitungen des 17. bis 20. Jahrhunderts. Hrsg. von Heinz-Dietrich Fischer. Pullach 1972 (Publizistisch-Historische Beiträge 2). S. 25-40, hier S. 38. 
Königlich privilegirte Berlinische Zeitung von Staats- und gelehrten Sachen, Vossische Zeitung, Gesellschaft mit beschränkter Haftung. Bezahlen aber mussten die Ullsteins Berlins älteste Zeitung erst einmal nicht.

Ullstein hatte in den Kaufvertrag eine Klausel einfügen lassen, dass im Falle eines europäischen Krieges die Zahlungen verschoben werden sollen. ${ }^{73}$ Am 1. Januar 1914 erfolgte die Übernahme der Vossischen Zeitung, es war der Beginn eines langwierigen Rettungsversuchs. ${ }^{74}$ Genau acht Monate später brach der Erste Weltkrieg aus. „Ein Schutzengel muss uns beigestanden haben“, erinnerte sich Hermann Ullstein im Exil. ${ }^{75}$ Doch gleich mit dem Übergang der Voss in Ullstein-Besitz mussten die neuen Eigentümer investieren, so wurde das Blatt einer gründlichen optischen Überarbeitung unterzogen: Unter der Leitung Georg Bernhards frischte man das Layout auf; neue Schrifttypen wurden integriert, die monotone Spaltenanordnung aufgehoben. ${ }^{76}$

Bei Ausbruch des Ersten Weltkriegs stellt sich der Ullstein-Verlag somit als Unternehmen mit der später in der Publizistik üblichen konzerninternen Mischfinanzierung dar: Das Geld machten massentaugliche Eigengründungen wie die Berliner Morgenpost; hoch angesehene, finanziell aber belastete Objekte wie die Vossische Zeitung wurden dem Portfolio durch Ankauf hinzugefügt. ${ }^{77}$

Der Informationsbedarf erreichte im Ersten Weltkrieg einen weiteren Höhepunkt und ließ die Auflagenhöhen, vor allem der Tageszeitungen, deutlich ansteigen. ${ }^{78}$ Das Lesebedürfnis der Bevölkerung nahm kontinuierlich zu, trotz zu-

73 Vgl. Der Spiegel (04/1952), Gott, S. 15.

74 Siehe dazu Heuss, Theodor: Drei Jahrhunderte Vossische Zeitung. In: Hundert Jahre Ullstein 1877-1977. Band 2. Hrsg. von Joachim W. Freyburg u. Hans Wallenberg. Frankfurt a. M./Berlin/Wien 1977. S. 119-140, hier S. 137.

75 Vgl. Hermann Ullstein, Haus Ullstein, S. 126.

76 Hierzu Klein, Michael: Georg Bernhard. Die politische Haltung des Chefredakteurs der Vossischen Zeitung 1918-1930. Frankfurt am Main/Berlin/Bern/New York/ Paris/ Wien 1999 (Europäische Hochschulschriften, Reihe 3: Geschichte und ihre Hilfswissenschaften 822). Rahmendaten Georg Bernhards bemerkenswerter Karriere: 1898 wurde er Handelsredakteur der Berliner Zeitung, kurz darauf der Berliner Morgenpost. Nach der Gründung einer kritischen Zeitung für Finanzwirtschaft (Plutus) schloss man Bernhard aus der SPD aus. Seit 1908 Verlagsdirektor bei Ullstein, kümmerte er sich bereits ab 1914 um die Ausrichtung der Vossischen Zeitung, deren Chefredakteur Bernhard 1920 wurde. 1924 trat er der DDP bei. Er blieb im Verlag bis 1930. 77 Stöber, Pressegeschichte, S. 259, vergleicht die Situation beim Ullstein-Verlag in dieser Hinsicht mit Cotta, dessen Allgemeine Zeitung nur durch das Morgenblatt für die Gebildeten Stände finanzierbar war.

78 Siehe Verhey, Jeffrey: The Spirit of 1914. Militarism, Myth, and Mobilization in Germany. Cambridge 2006 (Studies in the Social and Cultural History of Modern Warfare 10), S. 75. 
nehmender inhaltlicher Gleichförmigkeit. ${ }^{79}$ Die Auflage der Berliner Illustrirten Zeitung etwa überstieg in diesen Jahren erstmals die Millionengrenze. ${ }^{80}$

Trotz allem positionierte man sich politisch, auch und erst recht bei den Ullsteins. Seit ihrem Erwerb war die Vossische Zeitung dafür ausersehen, das maßgebende politische Organ des Verlags zu werden. ${ }^{81}$ Ihr Ankauf hatte dem Ullstein-Verlag die nötige Seriosität verschafft, um endgültig ernst genommen zu werden.

Der Verlag fuhr zwischen 1914 und 1918 eine zweigleisige Strategie. Neben der Tageszeitungs- und Zeitschriftensparte entwickelten die Ullsteins auch ein Konzept für eine Bücherreihe, die sich ganz auf das Themenfeld Weltkrieg fokussierte. In Ergänzung der Eine-Mark-Bücher wurden die Ullstein-Kriegsbücher entwickelt. ${ }^{82}$ Bereits im Herbst 1914 erschien mit Paul Oskar Höckers Kriegstagebuch aus Belgien und Frankreich mit dem Titel An der Spitze meiner Kompagnie mit einer Gesamtauflage von 399.627 Exemplaren. Berichte über Erlebnisse auf allen Kriegsschauplätzen folgten.

Der Technikbegeisterung des Zeitalters entsprechend, erzielten Flieger- und U-Boot-Geschichten die höchsten Auflagen dieser Reihe: Gunther Plüschows Der Flieger von Tsingtau (Band 23, Erscheinungsjahr 1916) verkaufte sich rund 610.000 Mal; Paul Königs Die Fahrt der ,Deutschland“ (Band 21, ebenfalls 1916 erschienen) fand 555.419 Leser und Manfred von Richthofens Der rote Kampfflieger (Band 30, Erscheinungsjahr 1917) 521.427 Käufer. Allein der zunehmende Papiermangel verhinderte noch höhere Auflagen: Der Papierpreis vervierfachte sich während des Krieges; Druck- und Fixierchemikalien kosteten nun das bis zu Sechsfache, Transport- und Expeditionskosten das Dreifache. ${ }^{83}$

Realistische Darstellungen, die den Kriegsalltag mit all seinen Schrecken und Widrigkeiten schilderten, hätten unter den Zensurbedingungen und der anhaltenden Kriegsbegeisterung ohnehin nicht erscheinen dürfen, auch ihre Leserzahl wäre wohl deutlich eingeschränkt gewesen: Die Heimat glaubte an den

79 Vgl. Creutz, Martin: Die Pressepolitik der kaiserlichen Regierung während des Ersten Weltkriegs. Die Exekutive, die Journalisten und der Teufelskreis der Berichterstattung. Frankfurt a.M./Berlin/Bern/New York/Paris/Wien 1996 (Europäische Hoschulschriften, Reihe III, Bd. 704).

80 Dazu Mendelssohn, Zeitungsstadt, S. 202.

81 Zur Rolle der Vossischen Zeitung in der Weimarer Republik siehe Sösemann, Bernd: Im Spiegel der Voß: Der Kampf um die Republik. In: Hundert Jahre Ullstein 1877-1977. Band 1. Hrsg. von Joachim W. Freyburg u. Hans Wallenberg. Frankfurt a. M./Berlin/Wien 1977. S. 217-264. Dazu auch: Koestler, Arthur: Als Zeuge der Zeit. Das Abenteuer meines Lebens. Bern/München 1983, S. 89.

82 Siehe Ullstein Verlag, 50 Jahre Ullstein, S. $89 \mathrm{ff}$.

83 Angaben nach Stöber, Pressegeschichte, S. 171. 
Sieg, sie wollte Heldengeschichten. Der Soldat im Feld wusste es besser, brauchte aber Ablenkung. Und Ullstein belieferte beide.

Am 19. April 1916 hatte der Reichskanzler die Zuteilung und Herstellung von Druckpapier einer „Kriegswirtschaftsstelle für das Zeitungsgewerbe $\mathrm{GmbH}$ “ übertragen. ${ }^{84}$ Sie setzte zweimonatlich das Papierkontingent neu fest. Gleichwohl bereitete diese Maßnahme eher kleinen Zeitungen und Verlagen Probleme. Die großen Verlage wie Ullstein verringerten einfach den Umfang. Da die Auflagen aufgrund des erhöhten Informationsbedarfs beim Publikum im Gegenzug trotzdem anstiegen, hielten sich die Verluste in Grenzen.

Auch privat schrammte die Familie Ullstein glücklich an Verlusten vorbei. Zwei Mitglieder der „Enkel-Generation“ und ein Neuankömmling, die allesamt ab den 1920er Jahren in das Verlagsgeschehen bis in die Zeit der Restitution eingreifen würden, wollten sich jetzt im Krieg beweisen. Heinz Ullstein, Jahrgang 1893 und der Sohn von Louis, war aufgrund „manifester Unterernährtheit“ als untauglich abgelehnt worden. ${ }^{85}$ Karl Ullstein, ebenfalls Jahrgang 1893 und der älteste Sohn von Hans, wurde bei den Kürassieren ausgebildet, diente dann aber bei der Feldartillerie. Karl Ullstein erhielt das Eiserne Kreuz, geriet allerdings 1917 in französische Kriegsgefangenschaft. Einer seiner Kriegskameraden war Fritz Ross, ein Österreicher schottischer Herkunft. ${ }^{86}$ Ross trat 1918 als Fachverlagsleiter bei Ullstein ein und heiratete Karls älteste Schwester Hilda. Ab 1929 saß Fritz Ross im Ullstein-Aufsichtsrat, er war es, der Remarques Im Westen nichts Neues ins Verlagsprogramm aufnahm.

Insgesamt waren im Ersten Weltkrieg 1.386 Angehörige des Unternehmens eingezogen worden. Bis 1921, dem Zeitpunkt also, als keine Gefangenen mehr zurückkehren konnten, ergab die Weltkriegsbilanz des Hauses Ullstein, dass 193 Mitarbeiter gefallen waren. Am 23. Februar 1926 wurden zum Andenken an die Gefallenen zwei Bronzetafeln im Treppenhaus des Ullstein-Hauses in der Kochstraße feierlich enthüllt. ${ }^{87}$

Wie wenig die Ullsteins sich von den politischen Umtrieben des Revolutionsnovembers 1918 beeindrucken ließen, unterstreicht ein Kauf, den sie in diesen Tagen tätigten: Sie erwarben ein großes Reservegrundstück (23.670 qm) an der Grenze zwischen Tempelhof und Mariendorf, zwischen Teltowkanal, Burggrafenstraße (der späteren Ullsteinstraße) und Berliner Straße (deren Südteil ab der Stubenrauchbrücke später in Mariendorfer Damm umbenannt wurde). We-

84 Siehe Koszyk, Kurt: Deutsche Presse 1914-1945. Geschichte der deutschen Presse, Teil III. Berlin 1972 (Abhandlungen und Materialien zur Publizistik 7), S. 23.

85 Hierzu: Nadolny, Ullsteinroman, S. 281.

86 Nadolny, Ullsteinroman, S. 290.

87 Nach: Ullstein Verlag, 50 Jahre Ullstein, S. $87 \mathrm{f}$. 
nige Jahre später, als die Inflation überstanden war, begannen hier die Planungen für das Druckhaus Tempelhof. ${ }^{88}$ Es sollte das einzige nennenswerte Gebäude des Ullstein-Konzerns sein, das den Zweiten Weltkrieg überstehen würde.

\subsection{Aufschwung an die Weltspitze und zweiter Berliner Zeitungskrieg}

Zwischen 1919 und 1929 stieg die Einwohnerzahl Berlins auf 4,3 Mio. an, nur New York und London waren noch größer. Über 60 \% der Einwohner waren erwerbstätig, diese hohe Quote resultierte aus der Tatsache, dass auch über $40 \%$ der Berlinerinnen einer Arbeit nachgingen. Ein Viertel des gesamten deutschen Aktienkapitals war in der Hauptstadt konzentriert. ${ }^{89}$ Und: Zwischen 1881 und 1932 stieg die Anzahl der in Deutschland herausgegebenen Zeitungstitel von etwa 2.400 auf über 4.700 an. ${ }^{90}$ Die insgesamt 93 mindestens sechsmal pro Woche erscheinenden Zeitungen erzielten die höchste Auflage der Welt. ${ }^{91} 1928$ wurden in der gesamten Republik 3.356 verschiedene Tageszeitungen herausgegeben - davon 147 in Berlin. Addiert man zu den Zeitungen noch die Zeitschriften, wurden in der deutschen Hauptstadt insgesamt 2.633 Publikationen produziert. $^{92}$

Seit die fünf Ullstein-Brüder kurz vor der Jahrhundertwende das Ruder im Verlag übernommen hatten, entwickelte sich ihr Unternehmen zum größten seiner Art in Europa. Auf mehreren Gebieten schufen die Inhaber die Prototypen von Zeitungen, Zeitschriften und Buchreihen. ${ }^{93}$ So hatten die Ullsteins 1919 vom Georg-Müller-Verlag eine Reihe von Klassikerausgaben erworben und diesen Ankauf zum Grundstock für eine Spezialabteilung gemacht, die mit Bezug auf die Goethe-Propyläenausgabe den Namen „Propyläen-Verlag“ erhielt. Nach deren großem Erfolg - der auch ein qualitativer war, da mit dieser Reihe die Ull-

88 Dazu: Bannehr, Eule, S. 17.

89 Angaben nach Mendelssohn, Zeitungsstadt, S. $360 \mathrm{f}$.

90 Hierzu Georgii, Eberhard: Zur Statistik der deutschen Zeitungen. In: Handbuch der deutschen Tagespresse. Berlin 1932, S. 18.

91 Vgl. Meier, Gerd: Die Regionalpresse der Weimarer Republik - Innovationen und Traditionen. In: Politischer Journalismus, Öffentlichkeiten und Medien im 19. und 20. Jahrhundert. Hrsg. von Clemens Zimmermann. Ostfildern 2006 (Schriften der Siebenpfeiffer-Stiftung 8). S. 169-192, hier S. 178.

92 Mendelssohn, Zeitungsstadt, S. 427.

93 Ausführliche Zusammenfassung erstellt für den High Commissioner for Germany (HICOG) am 04.01.1952, in: AS-UA, Bestand Ullstein, Band 22. 
steins erfolgreich gegen das Trivial-Image ihrer Bücher ankämpften ${ }^{94}$ - wurden in den folgenden Jahren weitere Buchreihen ähnlich Stils initiiert, etwa die Propyläen-Kunstgeschichte (1923-1929), das vierbändige Weltreich der Technik (1923 ff.) oder die Klassiker des Altertums. ${ }^{95}$

Mittlerweile belegte der Ullstein-Verlag im Berliner Zeitungsviertel mit seinen Büros drei Straßenzüge (Kochstraße 22-26, Charlottenstraße 9-12 und Markgrafenstraße 66-73a). Allein in Berlin wurden bis 1926 insgesamt 75 Filialen eingerichtet, womit der Markenname Ullstein im Straßenbild noch präsenter wurde. ${ }^{96}$ Ein Jahr später zählte der Konzern 14 Abteilungen, in denen anhand verschiedenster Maßnahmen auf die Diversifizierung des Publikums reagiert wurde. ${ }^{97}$

1920 gründete Ullstein gemeinsam mit der Film-Produktionsfirma Decla Bioscop die Uco-Film GmbH. Hier sollte eine perfekte Verwertungskette geschaffen werden: Ein literarischer Stoff, der zunächst als Fortsetzungsroman in einer der Ullstein-Zeitungen erfolgreich bekannt gemacht worden war, sollte danach als Buch erscheinen und schließlich auch noch auf die Leinwand gebracht werden. ${ }^{98}$ Immerhin fünf Filme wurden auf diese Weise bis 1923 tatsächlich produziert, darunter waren Fritz Langs Dr. Mabuse, der Spieler (zwei Teile 1921/22) oder F. W. Murnaus Phantom (1922). ${ }^{99}$ All diese neuen Entwicklungen erforderten auch eine Änderung in der Firmenstruktur.

Am 10. Januar 1921 wurde die offene Handelsgesellschaft Ullstein \& Co. in die Ullstein-Aktiengesellschaft umgewandelt. Sämtliche Aktien verblieben im Besitz der fünf Ullstein-Brüder, sie hatten diese durch einen unter sich abge-

94 Hierzu Koszyk, Der jüdische Beitrag, S. 212.

95 Schilderung nach Ullstein Verlag, 50 Jahre Ullstein, S. $106 \mathrm{f}$.

96 Siehe Dorn, Margrit u. AndreasVogel: Geschichte des Pressevertriebs in Deutschland. Mit einem Schwerpunkt auf der Entwicklung des Pressehandels. Baden-Baden 2001 (Stiftung Presse-Grosso 2), S. 103.

97 Vgl. Oels, David u. Ute Schneider: Masse, Mobilität, Moderne - Zur Einleitung. In: „Der ganze Verlag ist einfach eine Bonbonniere“. Ullstein in der ersten Hälfte des 20. Jahrhunderts. Hrsg. von David Oels u. Ute Schneider. Berlin/München/Boston 2015 (Archiv für Geschichte des Buchwesens 10). S. 1-18, hier S. 8.

98 Zur Verwertungskette bei Ullstein siehe auch: Podewski, Madleen: Wie der Ullstein Verlag „Werk“ und „Autor“ in Bewegung versetzt. Zu Vicki Baums „Menschen im Hotel“. In: „Der ganze Verlag ist einfach eine Bonbonniere“. Ullstein in der ersten Hälfte des 20. Jahrhunderts. Hrsg. von David Oels u. Ute Schneider. Berlin/München/Boston 2015 (Archiv für Geschichte des Buchwesens 10). S. 207-221.

99 Dazu Schüler, Bernhard: Der Ullstein-Verlag und der Stummfilm. Die Uco-Film GmbH als Ausdruck einer innovativen Partnerschaft. Wiesbaden 2013 (Mainzer Studien zur Buchwissenschaft 23). 
schlossenen Syndikatsvertrag dauerhaft an die Familie gebunden. ${ }^{100}$ Die Aktienanteile staffelten sich nach dem Alter der Brüder: Hans und Louis hielten jeweils $27 \%$, Franz $18 \%$, Rudolf $16 \%$ und Hermann $12 \%$.

Das Stammkapital betrug 20 Mio. Mark, drei der Brüder traten in den Aufsichtsrat ein: Hans als Vorsitzender, Louis als stellvertretender Vorsitzender und Rudolf. Franz übernahm den Vorstandvorsitz, Hermann wurde sein Stellvertreter. Allein zeichnungsberechtigt war Dr. Franz Ullstein. ${ }^{101}$

Womit sich auch die hausinternen Konfliktlinien der folgenden Jahre abzeichneten. Während sich Hans und Louis schon aus Altersgründen mehr und mehr vom Tagesgeschäft entfernt hielten und zunehmend die dritte Ullstein-Generation - etwa Karl Ullstein, Heinz Ullstein oder Fritz Ross - ans Ruder lassen wollten, verschrieb sich Rudolf ganz der Technik. Und es kam zu einem Bruch zwischen Franz und Hermann, der sich langsam vertiefte. ${ }^{102}$

Dominierten in den Weimarer Jahren zunächst die Kämpfe zwischen den beiden Vorständen um die Macht im Verlag - ein Kampf, den Franz für sich entschied -, so ging es am Ende der 1920er Jahre um die Haltung des Verlags zum aufkommenden Nationalsozialismus (siehe Kapitel 2.5). Hermann Ullstein, der sich deutlicher als seine Brüder gegen die „Braunhemden“ positionieren wollte, sagte über Franz: „Ihm war es zu verdanken, dass die Tageszeitungen und die Buchproduktion derart expandierten. [...] Im Verlag beanspruchte er eine beinahe diktatorische Macht, wie mein Bruder Louis und ich ihm unterstellten. Für länger als ein Jahrzehnt gab es deshalb erbitterte Fehden - aber sie hinderten den Verlag keineswegs daran, bis zur Spitze emporzusteigen. “"103

Dass die Zusammenarbeit zwischen den Brüdern in Krisensituationen dennoch funktionierte, unterstreichen die Maßnahmen, die die beiden Vorstände im Zuge der Inflation 1923 ergriffen.

Die galoppierende Geldentwertung ist für ein publizistisches Unternehmen noch schwieriger als für Firmen anderer Branchen, da man in anderen Gewerbezweigen zur Goldrechnung übergehen konnte; bei Zeitungen war das unmöglich, und so stellte die Inflation die bis dahin schwerste Krise für die Ullstein AG dar. Die Preise der Zeitungen und Zeitschriften mussten zunächst von Monat zu Monat, dann von Woche zu Woche und schließlich für jeden Tag neu kalkuliert werden.

100 Nach Ullstein Verlag, 50 Jahre Ullstein, S. 135.

101 Vgl. Koszyk, Deutsche Presse 1914-1945, S. 252.

102 Siehe Nadolny, Ullsteinroman, S. $383 \mathrm{f}$.

103 Nach Ullstein, Hermann: Brüder über Brüder. Hermann Ullstein erzählt aus der Familie. In: Hundert Jahre Ullstein 1877-1977. Band 1. Hrsg. von Joachim W. Freyburg u. Hans Wallenberg. Frankfurt a. M./Berlin/Wien 1977. S. 121-124, hier S. 123. 
Für die Auflagen der Zeitschriften und Zeitungen bedeutete die Inflationszeit oftmals eine negative Entwicklung ${ }^{104}$, so sank die Anzahl der verkauften Exemplare der Berliner Illustrirten von 1,5 Mio. auf 300.000. Die Illustrirte begann ihren Druck am Montag, kam aber erst am Donnerstag in den Handel. Da bei einer raschen Geldentwertung drei Tage einen finanziell kaum zu kalkulierenden Zeitraum darstellen, musste man den Preis, der auf den Kopf der Zeitung gedruckt wurde, raten - und lag oft falsch. ${ }^{105}$ Der Preis der Berliner Illustrirten Zeitung belief zu Beginn der Inflation auf zehn Pfennige, am Ende, im November 1923, kostete sie genau eine Billion Mark. Nach der Stabilisierung der Währung wurde der Preis auf 20 Pfennige festgesetzt. ${ }^{106}$

Zumindest für den Zwischenhandel ging man während der Inflationsphase dazu über, in Goldpfennigen zu rechnen. ${ }^{107}$ Der Wertverlust zwischen der Ankunft der Wäschekörbe voller Geld in der Zentrale bis zur Einzahlung der Noten bei der Bank war zu groß. Franz und Hermann Ullstein mussten sich also um Deviseneinnahmen bemühen, sie beschlossen, Schnittmuster, Kataloge und Magazine nicht nur in Deutsch, sondern in 12 Sprachen zu drucken. Schweizer Franken, US-Dollar und andere harte Währungen flossen auf diese Weise dem Verlag zu und trugen entscheidend dazu bei, dass Ullstein in der Inflationsphase sämtliche Sachwerte retten konnte. ${ }^{108}$

Und noch eine Maßnahme ermöglichte unverhoffte Einnahmen: Mit der galoppierenden Geldentwertung wuchs der Bedarf nach Zahlungsmitteln, dem die Reichsdruckerei nicht mehr nachkommen konnte. Alle leistungsfähigen Druckereien Berlins wurden nun Tag und Nacht mit dem Drucken von Geld beschäftigt, auch bei Ullsteins wurden Notendruckmaschinen aufgestellt und angeworfen. ${ }^{109}$ Diese Aufträge waren sehr begehrt, da die Reichsbank die Bezahlung für den Notendruck teilweise in Goldmark leistete.

Die Inflation hatte der Ullstein-Verlag in einem befriedigenden Zustand überstanden, nun blickte man nach vorn. Quasi ein Vorläufer der Zeitungsgrün-

104 In den Jahren der Hochinflation haben etwa 400 bis 500 deutsche Zeitungen ihren Betrieb eingestellt, vgl. Gossel, Medien und Politik, S. 197.

105 Hierzu Mendelssohn, Zeitungsstadt, S. 366.

106 Hermann Ullstein erinnert sich: „Denn da wir der Kaufkraft der neuen Währung (Rentenmark) nicht über den Weg trauten, verdoppelten wir den Preis von 10 Pfennig. Im Übrigen war der Anzeigenteil so enorm angewachsen, dass wir auch den redaktionellen Teil entsprechend vergrößern mussten, damit kein Ungleichgewicht entstand.“ Bei Ullstein, Haus Ullstein, S. 181. 107 Nach Ullstein Verlag, 50 Jahre Ullstein, S. 104.

108 In: Der Spiegel (04/1952), Gott, S. 15.

109 „In unserer Druckerei wurden nicht mehr Zeitungen, sondern Geldnoten gedruckt. Alle Zugänge mussten verschlossen werden und Beamte der Reichsbank standen Wache. Um die Maschinen herum saßen ältere Frauen und starrten fasziniert auf die Stelle, wo die fertigen Scheine herausflossen.“ Erinnerung Hermann Ullsteins in Ullstein, Haus Ullstein, S. 179. 
dungen des Verlags in Weimars stabilen Jahren war die Kinderzeitschrift Der heitere Fridolin - 1921 in den Handel gebracht, erreichte sie bald 300.000 Abonnenten. ${ }^{110}$ Da hier aber konsequent auf Anzeigen verzichtet wurde, erwies sich die im Zwei-Wochen-Rhythmus erscheinende Kinderzeitschrift als finanzieller Fehlschlag.

Weitaus erfolgreicher waren die Neugründungen der Folgejahre: 1924 kam das Magazin Uhu, 1925 die Zeitschrift Die Koralle und 1927 die Grüne Post heraus. Die Koralle hatte sich auf die populärwissenschaftliche Darstellung von Technik und Naturwissenschaft spezialisiert, sie pendelte sich rasch bei einer Auflage von etwa 60.000 Exemplaren ein. Der Uhu hingegen, eine Entwicklung Hermann Ullsteins, war ein literarisches Magazin, stets bemüht, mit den besten zeitgenössischen Autoren zu arbeiten, dazu gehörten Kurt Tucholsky (alias Peter Panter/Theobald Tiger), Vicky Baum, Walter Benjamin, Bertolt Brecht, Hermann Hesse, Erich Kästner, Else Lasker-Schüler, Thomas und Heinrich Mann usw. ${ }^{111}$ Im Jahr 1929 erreichte der Uhu eine Auflage von 200.000 Exemplaren, sie sank im Zuge der Weltwirtschaftskrise bis 1933 auf 110.000 Stück. Zum 1. September 1934 wurde der Uhu eingestellt. ${ }^{112}$

Die Grüne Post war eine Sonntagszeitung, konzipiert ursprünglich für die Landbevölkerung (es gab u. a. Landwirtschafts- und Jagdrubriken), fand sie auch in den Städten eine überraschend große Leserschaft. Im Verlag selbst ob ihrer wenig spektakulären Konzeption umstritten ${ }^{113}$, überschritt die Auflage der Grünen Post nach einem Jahr die Grenze von 500.000 Exemplaren, nach gut

110 Ausführlich bei: Elsberg, Heinz: Mit Kinderaugen gesehen. In: Hundert Jahre Ullstein 1877-1977. Band 1. Hrsg. von Joachim W. Freyburg u. Hans Wallenberg. Frankfurt a. M./Berlin/Wien 1977. S. 333-358, hier S. 336.

111 Über die neue Bildsprache und die damit einhergehende Wahrnehmung der Moderne vgl.: Rössler, Patrick: Zwischen „Neuem Sehen“ und der bildpublizistischen Massenware. Der Aufstieg des Fotojournalismus in Uhu, Querschnitt und Berliner Illustrirte Zeitung. In: „Der ganze Verlag ist einfach eine Bonbonniere“. Ullstein in der ersten Hälfte des 20. Jahrhunderts. Hrsg. von David Oels u. Ute Schneider. Berlin/München/Boston 2015 (Archiv für Geschichte des Buchwesens 10). S. 287-319.

112 Siehe zur Entwicklung des Magazins: Noack-Mosse, Eva: Uhu. In: Hundert Jahre Ullstein 1877-1977. Band 2. Hrsg. von Joachim W. Freyburg u. Hans Wallenberg. Frankfurt a. M./Berlin/ Wien 1977. S. 177-208, hier S. 185.

113 Die Grüne Post ging auf eine Initiative Louis Ullsteins zurück. Hermann, der eigentlich für den Zeitschriftenbereich verantwortlich war, zeigt sich wenig angetan. Nach der ersten Ausgabe sprach Hermann den Chefredakteur der Grünen Post, Richard Katz, an: „Wenn ich in der Eisenbahn ,Die Grüne Post‘ lesen und mich einer fragen sollte, ob sie in meinem Verlag erscheint, werde ich mich schämen. “ Nach Katz, Richard: Die Grüne Post. In: Hundert Jahre Ullstein 1877-1977. Band 2. Hrsg. von Joachim W. Freyburg u. Hans Wallenberg. Frankfurt a. M./ Berlin/Wien 1977. S. 167-176, hier S. $174 \mathrm{f}$. 
zwei Jahren erstmals die Millionengrenze. ${ }^{114}$ Im dritten Quartal 1933 hatte sie noch über 740.000 Leser. ${ }^{115}$ Die Zeitschrift spielte eine wichtige Rolle bei den Verhandlungen zum Zwangsverkauf 1934 (siehe Kapitel 3.2).

Die elitäre Monatszeitschrift Der Querschnitt, eine Gründung des Kunsthändlers Alfred Flechtheim mit Beiträgen in Englisch, Französisch und Italienisch, kam 1924 zum Ullstein-Verlag. Binnen zwei Jahren stieg die Auflage auf immerhin 13.000 Exemplare, 1931 lag sie bereits bei etwa 25.000. ${ }^{116}$ Das waren für Ullstein-Verhältnisse eher vernachlässigbare Größen, gleichwohl gehörte das Magazin, was Geschmack und Lebensstil betraf, zu den führenden Publikationen der Weimarer Republik. Der Querschnitt war eines der treffendsten Spiegelbilder der 1920er Jahre. ${ }^{117}$ Im April 1933 erschien das Magazin zum letzten Mal im Ullstein-Verlag, dann kurzzeitig beim Kurt Wolff-Verlag, Berlin. Sein letztes Auffangbecken war in den Jahren 1935 und 1936 der speziell für den Querschnitt gegründete Heinrich Jenne-Verlag in Berlin-Steglitz.

Zwei Tageszeitungen, bei denen die Ullsteins das Innovationspotenzial ausgeschöpft sahen, wurden 1922 vereinigt: Die Berliner Abendpost, von den Ullsteins 1887 als Zeitung Berlins für das provinzielle Reichsgebiet gegründet, wurde mit der Berliner Allgemeinen Zeitung, einem im Jahr 1909 erworbenen Blatt, zusammengeführt. Im September 1928 wagte Ullstein eine spektakuläre Neugründung: Mit Tempo hatte man eine Tageszeitung im kompakten Berliner Format für die jüngeren Leser der Nachkriegsgeneration konzipiert ${ }^{118}$; mit ihrer Gründung reagierte Ullstein auf den Ankauf des Acht-Uhr-Abendblatt durch den Mosse-Verlag. Dem schnellen Lebensgefühl der Weimarer Jahre entsprach nicht nur der Tempo-Titel ${ }^{119}$, sondern auch ihre Erscheinungsweise: Dreimal täglich kamen verschiedene Ausgaben auf den Markt, alle zwischen 16 und 19 Uhr. ${ }^{120}$

114 Vgl. Wentzel, Fritz: Schnelligkeit ist immer Trumpf. Vertrieb im Hause Ullstein/Axel Springer. In: Hundert Jahre Ullstein 1877-1977. Band 3. Hrsg. von Joachim W. Freyburg u. Hans Wallenberg. Frankfurt a. M./Berlin/Wien 1977. S. 43-74, hier S. 71.

115 Vgl. Auflagenzahlen der Ullstein-Blätter 1933, in: Freyburg, Joachim W. u. Hans Wallenberg (Hrsg.): Hundert Jahre Ullstein 1877-1977, Frankfurt a. M./Berlin/Wien 1977, Band 1-4, Band 2, S. $256 \mathrm{f}$.

116 Hierzu Mendelssohn, Zeitungsstadt, S. 373.

117 Zur Rolle des Magazins vgl. u.a. Hering, Gerhard F.: Der Querschnitt. In: Hundert Jahre Ullstein 1877-1977. Band 2. Hrsg. von Joachim W. Freyburg u. Hans Wallenberg. Frankfurt a. M./Berlin/Wien 1977. S. 209-255.

118 Eine der Innovationen von Tempo war die Einführung der aktuellen Bildberichterstattung. Siehe hierzu: Macias, José: Die Entwicklung des Bildjournalismus. München u.a. 1990, S. 31 f. 119 Fulda, Bernhard: Press and Politics in the Weimar Republic. Oxford 2009, S. 35, weist darauf hin, dass Tempo am rechten publizistischen Rand bald als Synonym für die „Amerikanisierung der Presse“ und als „Asphaltblüte“ (Der Jungdeutsche vom 13. September 1928) oder unverhohlen antisemitisch als „jüdische Hast“ diffamiert wurde. Siehe hierzu auch: Escher, 
Hier lag sicher auch einer der Gründe, warum Tempo nie an den wirtschaftlichen Erfolg der anderen Tageszeitungen des Ullstein-Verlags anknüpfen konnte $^{121}$ : Zum einen fiel in diesem kurzen Zeitfenster nicht genug Material an, um die drei Ausgaben sinnvoll auch nur ansatzweise mit verschiedenen Inhalten zu füllen; zum anderen überschnitt sich Tempo mit den bereits etablierten Berliner Abendzeitungen. Zudem mangelte es ihr an Anzeigenkunden. ${ }^{122}$

Dabei war sie durchaus kostenintensiv: In den ersten Jahren ihres Erscheinens wurden für die Fotos und Illustrationen der Tempo mehr ausgegeben als bei jeder anderen Ullstein-Tageszeitung. ${ }^{123}$ Ein besonderer Schwerpunkt lag neben der Börsen- und Sportberichterstattung auf populärwissenschaftlichen Berichten und Fortsetzungsromanen, Kurt Tucholsky und Erika Mann gehörten zu den ständigen Tempo-Autoren. Wie bei allen Boulevard-Blättern aber waren es die sensationsheischenden Geschichten, die über Auflagengewinne oder -verluste entschieden, so auch bei Tempo: Die Zeitung profitierte deutlich vom (und befeuerte den) sogenannten „Sklarek-Skandal“, einem kommunalpolitischen Korruptionsprozess im Jahr 1929. Die Firma Sklarek belieferte die städtische Verwaltung mit Dienstkleidung und hatte jahrelang erfolgreich zahlreiche gefälschte Rechnungen eingereicht, der Gesamtschaden betrug etwa 10 Mio. RM. ${ }^{124}$ Tempo konnte mit der anti-demokratischen Grundstimmung des Skandals auflagentechnisch deutlich Boden gutmachen. ${ }^{125}$

Clemens: Judenpresse. In: Handbuch des Antisemitismus. Judenfeindschaft in Geschichte und Gegenwart. Band. 3: Begriffe, Theorien, Ideologien. Hrsg. von Wolfgang Benz. Berlin/New York 2010, S. 156-157.

120 Vgl. Wentzel, Schnelligkeit, S. 72.

121 Die verkaufte Auflage von Tempo lag 1929 bei 118.500 Exemplaren.

122 Siehe Tribukait, Maren: Zwischen „berechtigter Sensation“ und zu viel „Tempo“. Zum Unterhaltungsjournalismus des Ullstein Verlags. In: „Der ganze Verlag ist einfach eine Bonbonniere“. Ullstein in der ersten Hälfte des 20. Jahrhunderts. Hrsg. von David Oels u. Ute Schneider. Berlin/München/Boston 2015 (Archiv für Geschichte des Buchwesens 10). S. 344-361, hier S. 355.

123 Vgl. Hung, Jochen: „Die Zeitung der Zeit“. Die Tageszeitung Tempo und das Ende der Weimarer Republik. In: „Der ganze Verlag ist einfach eine Bonbonniere“. Ullstein in der ersten Hälfte des 20. Jahrhunderts. Hrsg. von David Oels u. Ute Schneider. Berlin/München/Boston 2015 (Archiv für Geschichte des Buchwesens 10). S. 137-159, hier S. 139.

124 Siehe Reese, Dagmar: Skandal und Ressentiment. Das Beispiel des Berliner Sklarek-Skandals von 1929. In: Anatomie des politischen Skandals. Hrsg. von Rolf Ebbinghausen u. Sighard Neckel. Frankfurt/M. 1989. S. 374-395.

125 So Fulda, Press and Politics, S. 37: „Tempo established itself as one of the most vociferous prosecutors, and attracted a lot of attention by its sensationalist exposure of local corruption. Very different from its previous policy of almost abstaining from political coverage, and contrary to Ullstein's long tradition of supporting the democratic cause, the ,Tempo now joined Hugenberg’s ,Nachtausgabe‘ and Münzenberg’s ,Welt am Abend‘ in attacking Berlin's political 
Nur unterbrochen durch die Inflationsmonate, steigerten die deutschen Zeitungen und Zeitschriften grundsätzlich während der 1920er Jahre kontinuierlich ihre Auflagen. ${ }^{126}$ Damit einher ging eine deutliche Verschärfung des Berliner Zeitungsmarkts. Für den Ullstein-Verlag bedeutete dies massive Konkurrenz, vor allem durch den Scherl-Verlag unter Alfred Hugenberg.

Hugenberg führte als Gründungsmitglied den Alldeutschen Verband, von 1909 bis 1918 leitete er das Finanzwesen der Friedrich Krupp AG. 1918 gehörte er zu den Mitgründern der Deutschnationalen Volkspartei (DNVP). ${ }^{127}$

Als der kinderlose August Scherl 1913 Reichskanzler Bethmann-Hollweg informierte, dass er die Stammanteile an seinem Verlag verkaufen würde - Rudolf Mosse soll ihm dafür 11,5 Mio. Mark geboten haben, auch die Ullsteins hatten zum Entsetzen des Kaisers ihr Interesse an dem Konkurrenzunternehmen bekundet $^{128}$-, erklärte er zudem, dass er seine national-konservativ ausgerichteten Zeitungen lieber an regierungstreue Unternehmer veräußern würde. Der Scherl-Konzern wurde 1914 von einem 59-köpfigen Konsortium namens Deutscher Verlagsverein übernommen. ${ }^{129}$ Hugenberg wiederum übernahm 1916 die Schulden jenes Vereins, unter der Voraussetzung, dass er die Leitung des Scherl-Verlags von nun an innehatte, er wurde Vorsitzender des Aufsichtsrates. Im selben Jahr errichtete er mit der Allgemeinen Anzeigen GmbH (Ala) eine Firma zur Vermittlung von Zeitungswerbung.

Im September 1922 gründete Hugenberg mit der Wirtschaftsstelle der Provinzpresse (Wipro) einen eigenen Materndienst, der sich vor allem auf die Provinzpresse im deutschen Osten spezialisierte. Bis 1924 belieferte die Wipro bereits über 1.000 Zeitungen, das entsprach $35 \%$ aller deutschen Blätter. ${ }^{130}$ Noch während des Weltkriegs 1913, hatte Hugenberg die Telegraphen-Union GmbH

leadership. Although the emphasis lay on sensationalist revelations and was not driven by an anti-democratic Weltanschauung, effectively the ,Tempo' contributed to the growing number of voices denigrating the democratic system.“

126 Ausführliche Analyse bei Fulda, Press and Politics, S. $13 \mathrm{f}$.

127 Hierzu Fischer, Heinz-Dietrich: Alfred Hugenberg (1865-1951). In: Deutsche Presseverleger des 18. bis 20. Jahrhunderts. Hrsg. von Heinz-Dietrich Fischer. Pullach 1975 (Publizistisch-Historische Beiträge 4). S. 295-308, hier S. $295 \mathrm{f}$.

128 Siehe Gossel, Medien und Politik, S. 253.

129 Die Begründung des Vereins, so erläuterte es eine Stellungnahme des Innenministers von Dallwitz, lag „im dringenden staatlichen Interesse. Ohne die Opferwilligkeit der Vereinsmitglieder wäre der Scherlsche Verlag unter demokratische Leitung gelangt. Es wären mithin diejenigen Berliner Zeitungen, die in Folge ihrer großen Verbreitung für die Anschauungen der breiten Masse des Volkes von großer Bedeutung sind, ausnahmslos in staatsfeindliche Richtung geleitet worden." Nach Guratzsch, Dankwart: Macht durch Organisation. Die Grundlagen des Hugenbergschen Presseimperiums. Düsseldorf 1974, S. 279.

130 Vgl. Fischer, Hugenberg, S. 301. 
(T. U.), ein Zusammenschluss mehrerer kleiner Nachrichtenbüros, als Konkurrenz zu Wolffs Telegraphischem Bureau mitgegründet. ${ }^{131} 1919$ übernahm Hugenberg die Mehrheit an der T. U. und baute den Nachrichtendienst erheblich aus. Komplettiert zum Multi-Media-Unternehmen wurde der Konzern unter Hugenbergs Leitung 1927, als er sich die Stimmenmehrheit an der Universum Film AG, der Ufa, sicherte. ${ }^{132}$ Die unter seiner Leitung stehenden Firmen, allen voran die Publikationen des Scherl-Verlags, wiesen in den Jahren der Weimarer Republik zunächst eine rechtsnationale, später eine offen nationalsozialistische Ausrichtung auf.

Den beiden Tageszeitungen des Scherl-Verlags, dem Lokal-Anzeiger und dem Tag, wurden zwei Neugründungen hinzugefügt, die sich in ihrer Erscheinungsweise klar gegen die liberalen Ullstein-Publikationen richteten: der Montag und die Nachtausgabe. ${ }^{133}$ Zeitgleich jedoch versuchten noch mehr Protagonisten ihr Verlegerglück in Berlin, an den Auslageregalen der Kioske wurde es eng. Um 1925 gab es in der Hauptstadt 30 Tageszeitungen, hinzu kamen nochmals 30 bis 40 Stadtteil- und Bezirksblätter. ${ }^{134}$

Ullsteins B.Z. am Mittag wurde gleich mehrfach attackiert: Zunächst vom 12-Uhr-Blatt, das 1919 von ehemaligen Redakteuren des Acht-Uhr-Abendblatts (Mosse-Verlag) gegründet worden war - bei seinem ersten Erscheinungstag am 19. Januar konnte es mit der Sensation der Ermordung Luxemburgs und Liebknechts aufwarten. ${ }^{135}$ Genau wie die B.Z. am Mittag setzte das 12-Uhr-Blatt einen deutlichen Schwerpunkt bei Sportthemen. Scherls Nachtausgabe nun erschien ab 1923 stets gegen 16 Uhr, zu dem Zeitpunkt also, da die B.Z. am Mittag und das 12-Uhr-Tageblatt bereits überholt waren. Während der ersten Jahre ihres Erscheinens kam die Nachtausgabe kaum über eine verkaufte Auflage von 30.000 Exemplaren hinaus. Ab 1925 jedoch holte sie rasant auf, 1929 hatte sie mit einer Auflage von 200.000 die B. Z. am Mittag überflügelt.

Und wie sah es bei den Wochenzeitungen aus? Ullstein hatte im Mai 1920 die Montagspost, ein liberales Blatt im Stil der Berliner Morgenpost, gegründet. Gemeinsam mit Helmuth von Gerlachs demokratisch-pazifistischer Welt am Montag hatte die Montagspost den bisher zeitungsfreien ersten Wochentag - zu-

131 Um 1914/15 hatte auch Ullstein mit dem planmäßigen Aufbau eines Korrespondentennetzes begonnen, aus dem in den Folgejahren der „Ullstein-Nachrichtendienst“ erwuchs. 1927 belieferte der Dienst 15 deutsche und zehn ausländische Zeitungen. Vgl. Ullstein Verlag, 50 Jahre Ullstein, S. 129.

132 Mosse und Ullstein hatten zuvor eine Beteiligung an der defizitären Ufa abgelehnt, siehe Gossel, Medien und Politik, S. 272.

133 Nach Mendelssohn, Zeitungsstadt, S. $382 \mathrm{f}$.

134 Vgl. Fulda, Press and Politics, S. 17.

135 Siehe Mendelssohn, Zeitungsstadt, S. 383. 
mindest was wöchentliche Publikationen betraf - als Erscheinungstag besetzt. Dagegen nun gründete Hugenbergs Scherl-Verlag 1921 den Montag als „einzige rechtsstehende Montagszeitung der Hauptstadt“, so ihr Kopftitel. ${ }^{136}$ Der Montag erschien bereits Sonntagabend - das Berliner Zeitungsfeld war so eng besetzt, dass sich die Erscheinungszeiten immer weiter nach vorn verschoben. $\mathrm{Zu}$ diesen Konkurrenten trat 1923 noch der linksdemokratische Montag Morgen, kurz $M . M$. genannt.

In diesem erneuten Zeitungskrieg zwischen dem Ullstein-Verlag und den Scherl-Publikationen, nun unter der Führung Hugenbergs, war es dem liberalen Haus an der Kochstraße weiterhin möglich, sich zu behaupten. Zumindest bis zur Weltwirtschaftskrise konnte die Konkurrenz den Ullstein-Publikationen keine ernsthaften Schäden zufügen - zu vielfältig war die Berliner Presselandschaft, $\mathrm{zu}$ heterogen die Leserschaft. Im Ullstein-Verlag hielt man große Stücke auf die eigene politische Neutralität.

\subsection{Die Ullstein AG in der Weltwirtschaftskrise (1929-1933)}

Die Massennachfrage nach Unterhaltung nach dem Ersten Weltkrieg veränderte auch die Bedeutungsstruktur auf dem Berliner Zeitungsmarkt. ${ }^{137}$ Die traditionelle politische Elitenpresse geriet zunehmend publizistisch und finanziell in eine Abseitsstellung, zeitgleich setzte sich die Boulevardpresse in den Großstädten Deutschlands als führendes politisches Kommunikationsinstrument durch. Konkret für Berlin bedeutete das einen Anstieg des Marktanteils der Boulevardmedien von unter $10 \%$ zu Beginn der 1920er Jahre auf nahezu 40 \% am Ende des Jahrzehnts. ${ }^{138}$

Nur zwei der fünf Ullstein-Brüder waren politisch aktiv: Hans, der älteste, der sich inzwischen vom aktiven Tagesgeschäft zurückgezogen hatte und der viele Jahre als freisinniger Abgeordneter im Berliner Stadtparlament tätig war, und Hermann, der jüngste der Ullstein-Brüder, der $1918 \mathrm{zu}$ den Gründern der Deutschen Demokratischen Partei (DDP) gehörte, in die sechs Jahre später auch Georg Bernhard, der Chefredakteur der Vossischen Zeitung, eintrat. ${ }^{139}$ Im Gegensatz zu den Produkten der konkurrierenden Verlage trat die politische Präferenz

136 Hierzu Fulda, Press and Politics, S. 17.

137 Siehe Fulda, Bernhard: Die Politik der „Unpolitischen“. Boulevard und Massenpresse in den zwanziger und dreißiger Jahren. In: Medialisierung und Demokratie im 20. Jahrhundert. Hrsg. von Frank Bösch u. Norbert Frei. Göttingen 2006 (Beiträge zur Geschichte 20. Jahrhunderts 5). S. 48-72, hier S. 51.

138 Fulda, Politik der „Unpolitischen“, S. 52.

139 Siehe Koszyk, Deutsche Presse 1914-1945, S. 251. 
der Ullstein-Blätter deutlich weniger zutage. ${ }^{140}$ Es galt das Motto, dass die Berliner Morgenpost an ihrem ersten Erscheinungstag, dem 20. September 1898, ausgegeben hatte: „Parteinehmer - nicht Parteigänger“.

In den illustrierten Ullstein-Massenblättern versuchte man fast bis zum Ende der Republik zunächst eine unpolitische Linie zu wahren - das legendäre Foto von Friedrich Ebert in Badehose auf dem Titel der Berliner Illustrirten vom 21. August 1919 sollte tatsächlich nur die politische Zeitenwende widerspiegeln, erhielt jedoch unabsichtlich den Status einer anti-republikanischen Ikone. ${ }^{141}$ Aus der angestrebten neutralen Haltung im Hause Ullstein resultierte ein „Streben nach größtmöglicher Übereinstimmung mit dem Leserkreis“142, eine opportunistische Haltung, die nur selten durchbrochen wurde, und wenn, dann nicht bei den Massenmedien des Ullstein-Verlags. ${ }^{143}$

Am Ende der Weimarer Republik, als der politische wie der wirtschaftliche Druck stetig zunahm, erteilte die Ullstein-Verlagsleitung eine hausinterne Anweisung zum unpolitischen Verhalten seiner Massenblätter: „Weder die BZ, noch das Tempo, noch die Montagspost haben die Aufgabe, aktiv in den politischen Kampf einzugreifen." ${ }^{144}$ Im Dezember 1931 verlor Franz Höllering, der Chefredakteur der B.Z. am Mittag, seinen Posten, weil sich die Regierung Brüning über eine Titelgeschichte über Nationalsozialisten in dem von ihm verantworteten Blatt echauffiert hatte. ${ }^{145}$ Höllering, den die Ullsteins erst kurz zuvor

140 Ein Umstand, der gleichwohl erst mit dem - von den Ullstein-Publikationen mit Ausnahme der Vossischen bis an den Rand des Erträglichen neutral bewerteten - massiven Zulauf für die Nationalsozialisten ab 1928 und der damit einhergehenden Radikalisierung der Gesellschaft zu verlagsinternen Konflikten führte; anti-demokratische Tendenzen bekämpfte Ullstein durchaus seit Anbeginn der Weimarer Republik. Exemplarisch hierfür ist etwa die Ablehnung des Kandidaten Hindenburg für die Reichspräsidentenwahl (B.Z. am Mittag vom 8. März 1920); mehr Beispiele bei Asmuss, Burkhard: Republik ohne Chance? Akzeptanz und Legitimation der Weimarer Republik in der deutschen Tagespresse zwischen 1918 und 1923. Berlin/New York 1994 (Beiträge zur Kommunikationsgeschichte 3). S. 252.

141 Vgl. Birkenfeld, Wolfgang: Der Rufmord am Reichspräsidenten - Zu Grenzformen des politischen Kampfes gegen die frühe Weimarer Republik 1919-1925. In: Archiv für Sozialgeschichte 5 (1965). S. 453-500.

142 Zitiert nach Koszyk, Deutsche Presse 1914-1945, S. 251.

143 „One female Morgenpost reader explained she was quite satisfied that there is at least one newspaper which reports as good as nothing on politics'. Although she was not necessarily an accurate account of the Morgenpost's offerings, it reflected the widespread consensus that the entertainment provided by the Ullstein paper was the decisive buying factor“, so Fulda, Press and Politics, S. 27.

144 Rundschreiben vom 8. Dezember 1931, zitiert bei Fulda, Politik der „Unpolitischen“, S. 62. 145 So Fulda, Politik der „Unpolitischen“, S. 62. Der Titel der B. Z. am Mittag vom 14. Dezember 1931 lautete: „Wozu braucht Hitler Flugzeuge? Seine Organisation der S. A.-Flieger“. Diskutiert zudem bei: Eksteins, Modris: The Limits of Reason. German Democratic Press and the Collapse 
von Münzenbergs Arbeiter Illustrierte Zeitung abgeworben hatten, hatte sich bereits seit seinem Dienstantritt bei der B.Z. am Mittag aufgrund seiner deutlichen Berichterstattung über die Nationalsozialisten den Zorn der Regierung Brüning zugezogen. ${ }^{146}$ Deutlich stärker als die Ullstein-Zeitungen positionierten sich jedoch die Massenblätter des ebenfalls liberal-demokratischen Mosse-Verlags. ${ }^{147}$

Die Vossische Zeitung hatte unter Führung Georg Bernhards ab der Zeit der Kabinette Cuno und Stresemann zumindest eine deutliche Haltung gegen die Schwerindustrie und ihren faktischen Führer Hugo Stinnes eingenommen, bevor sie sich ab Herbst 1923 auch offen gegen Hugenberg wandte. ${ }^{148}$ Grundsätzlich verfolgte die Vossische Zeitung mit ihrer nationalliberalen Linie eine Unterstützung der Politik Gustav Stresemanns. ${ }^{149}$ Aber auch in ihrer Beurteilung des Nationalsozialismus zeigte sich die Vossische zumeist kompromisslos. ${ }^{150}$ Wobei die Zeitung „den künftigen deutschen Diktator“151 eher aus den Reihen der DNVP, also Hugenberg persönlich, erwartete.

Zusammenfassend herrschte demnach - mit Ausnahme bei der rechtsliberal eingestellten Vossische Zeitung - bei den Ullstein-Zeitungen eine zögerliche

of Weimar Democracy. Oxford 1975, S. 235-237. In der Weltbühne vom 05.01.1932 befasste sich auch Carl von Ossietzky mit der Entlassung des B.Z. am Mittag-Chefredakteurs: „Bei Ullsteins heißt das Ideal: ein ,Völkischer Beobachter' mit der Genehmigung des Rabbinats, von Brüning ebenso geschätzt wie von Braun und auch von den Kommunisten gern auf der Straße gekauft; ein Bastard von Goebbels und der Tante Voss. Da sich dies bizarre Verlagsideal nicht verwirklichen lässt, behilft man sich einstweilen mit einem reichlich chimärischen inneren Gleichgewicht; man dämpft, man retuschiert, man untersagt der ,Voss‘ etwa den Gebrauch des Wortes ,Nazi‘, um die Leute ,nicht unnütz zu reizen‘“ Nach Ossietzky, Carl von: Der Fall Franz Höllering. In: Die Weltbühne 1/28 (1932). Heft 1, S. 1-6. Zit. nach: Ossietzky, Carl von u. Werner Boldt (Hrsg.): Sämtliche Schriften. Bd. 6: 1931-1933. Hamburg 1994, S. 285-291.

146 Fulda, Press and Politics, S. 189 f., nennt exemplarisch die B.Z am Mittag-Schlagzeilen „Hitlers braune legale Armee“ (4. Dezember 1931) und „Hitler drängt sich in die Außenpolitik“ (5. Dezember 1931). Höllering wurde durch Fritz Stein, einen Korrespondenten des Hamburger Fremdenblatts, ersetzt, der unmittelbar nach seiner Berufung zum B.Z. am Mittag-Chefredakteur einen Brief an Brüning schrieb, in dem Stein - in Anbetracht der politischen Gründe für seine Berufung - versprach, der Zeitung nun eine neue politische Form zu geben.

147 Als Beispiel offeriert Fulda, Politik der „Unpolitischen“, S. 56, eine Titelgeschichte des Acht-Uhr-Abendblatts vom 13.09.1930, in der, graphisch unterstützt, die Schlagzeile „Moskau finanziert Nazis!“ lautete. Fulda fasst zusammen: „Tatsächlich profilierten sich das ,Acht UhrAbendblatt‘, Mosses ,Berliner Volks-Zeitung‘ und Münzenbergs ,Welt am Abend“ als schärfste publizistische Gegner des Nationalsozialismus bis 1933, besonders durch eine kontinuierliche Berichterstattung über die angeblich dunklen Quellen nationalsozialistischer Parteifinanzen.“

148 Siehe Klein, Georg Bernhard, S. $83 \mathrm{f}$.

149 Vgl. Koszyk, Der jüdische Beitrag, S. 205.

150 Zahlreiche Beispiele bei Koszyk, Deutsche Presse 1914-1945, S. 257 f.

151 Artikel „Der Jünger des Chaos“ in: Vossische Zeitung vom 22.09.1929, Nr. 227/1929. 
politische Haltung vor. ${ }^{152}$ Unangetastet jedoch war das Grundbekenntnis der Ullstein-Blätter zur republikanischen Demokratie. Gemäß der hausinternen Leitlinie, stets auf „gekonnten Unterhaltungs-Journalismus bedacht“ $\mathrm{zu}$ sein, wirkten die Publikationen unter dem Zeichen der Eule jedoch auf politischem Feld konfliktverdrängend. ${ }^{153}$ Allein bei seinem politischen Vorzeigeblatt war Ullstein also etwas mutiger, was politische Stellungnahmen und Versuche der Einflussnahme anging, als bei seinen Boulevardprodukten. ${ }^{154}$

Hermann Ullstein forderte seit 1928 als einziger der Brüder, die neutrale Haltung des Verlags zu überdenken - vergeblich. Als er sie überreden wollte, Richard Lehmann, den Chefredakteur der von Hermann eigenständig erworbenen Neuen Leipziger Zeitung, zum Chefredakteur der Berliner Morgenpost zu berufen, wurde dies von der Familie abgelehnt ${ }^{155}$ : Um die Auflagenhöhen nicht zu gefährden, könne man es sich, so sein Bruder Franz, „nicht leisten, eine politi-

152 Nichtsdestotrotz erzeugte der anwachsende ökonomische Druck immer wieder neue verlagsinterne Diskussionen über den Grad der Politisierung der Blätter, und zwar sowohl bei Mosse als auch bei Ullstein. Siehe hierzu: Requate, Jörg: Zwischen Profit und Politik. Deutsche Zeitungsverleger im ersten Drittel des 20. Jahrhunderts. In: Großbürger und Unternehmer. Die deutsche Wirtschaftselite im 20. Jahrhundert. Hrsg. von Dieter Ziegler. Göttingen 2000 (Beiträge zur europäischen Gesellschaftsgeschichte 17). S. 167-186, hier S. 184.

153 Vgl. Friedrich, Thomas: Die Berliner Zeitungslandschaft am Ende der Weimarer Republik. In: Berlin 1932. Das letzte Jahr der Weimarer Republik. Politik, Symbole, Medien. Hrsg. von Diethart Kerbs u. Henrick Stahr. Berlin 1992. S. 56-67, hier S. 63.

154 Auch die mit Abstand größte illustrierte Deutschlands, Ullsteins Berliner Illustrirte Zeitung, titelte äußerst selten mit realistischen, der Weltwirtschaftskrise geschuldeten Themen. Beispiele und Einschätzung bei Kerbs, Diethart: Die illustrierte Presse am Ende der Weimarer Republik. In: Berlin 1932. Das letzte Jahr der Weimarer Republik. Politik, Symbole, Medien. Hrsg. von Diethart Kerbs u. Henrick Stahr. Berlin 1992. S. 68-89, hier S. $82 \mathrm{f}$.

155 Die Neue Leipziger Zeitung (NLZ), die mit einer Auflage von 100.000 Exemplaren zweitgrößte Provinzzeitung der Weimarer Republik, erschien zwischen 1921 und 1940, bevor sie mit der Leipziger Tageszeitung zur Neuen Leipziger Tageszeitung vereinigt wurde, welche ihr Erscheinen 1945 einstellte. Auch während der NS-Jahre fungierte der von Hermann Ullstein geschätzte Richard Lehmann als „Hauptschriftleiter“, zeitweise war er Hauptmann einer Propaganda-Kompanie, bevor er 1940 fiel. Er ist nicht zu verwechseln mit seinem Namensvetter, dem sozialdemokratischen Widerstandskämpfer Richard Lehmann, der zeitweise Chefredakteur der Leipziger Volkszeitung war, der 1945 von den Nationalsozialisten hingerichtet wurde und nach dem heute eine Straße in der Leipziger Südvorstadt benannt ist. Siehe hierzu: Glosikova, Viera, Meißgeier, Sina u. Ilse Nagelschmidt (Hrsg.): „Ich träumte: ich saß in der Schule der Emigranten...“. Der jüdische Schriftsteller und Journalist Hans Natonek aus Prag. Berlin 2016 (Literaturwissenschaft 61), S. 78. Hermann Ullsteins NLZ war ursprünglich als politisch neutrales Blatt gegründet worden, als Mittlerin zwischen Bürgertum und Arbeiterparteien. Siehe hierzu: Meyen, Michael: Leipzigs bürgerliche Presse in der Weimarer Republik. Leipzig 1996, S. 128. 
sche Meinung zu haben [...] Unsere Stärke liegt darin, keine Meinung zu haben." ${ }^{156}$

Von den Diskussionen um die politische Ausrichtung unberührt blieb die wirtschaftliche Expansion der Ullstein AG. Das Ullstein-Imperium beschäftigte 1926 mit ca. 8.400 Mitarbeitern $15 \%$ aller Arbeitnehmer im Berliner Buchdruckund Zeitungsgewerbe. ${ }^{157}$ Eine weiterer Ausbau der Produktpalette wurde für die kommenden Jahre nicht ausgeschlossen und so entschied man sich im Verlag zu einer bedeutenden Investition: 1924 begannen die Planungen für das Druckhaus Tempelhof.

Nach den Entwürfen Eugen Schmohls errichtete man binnen zweieinhalb Jahren Europas größtes Druckhaus, wuchtig und kubisch, mit einem 13 Stockwerke aufragenden, 76 Meter hohen Turm. ${ }^{158}$ Bei der Konzeption entschied man sich für die Trennung von Tageszeitungsbetrieb (der samt Setzerei und Druckerei in der Kochstraße verblieb) und Zeitschriftendruckerei, die nun in Tempelhof angesiedelt wurde. Das neue Gebäude erstreckte sich auf 9.556 Quadratmetern Grundfläche, es gab sieben ober- und zwei unterirdische Stockwerke mit einer Gesamtnutzungsfläche von ca. 38.000 Quadratmetern. Auch die Repräsentation des Druckhauses ließ man sich einiges kosten, so wurden fast $10 \%$ der Bausumme für die Steinmetzarbeiten an der Fassade verwendet. ${ }^{159}$

Das Druckhaus Tempelhof war neben dem von Hans Poelzig errichteten Hochhaus der I. G. Farben-Werke in Frankfurt/Main der größte private Fabrikbau Deutschlands der Jahre 1925 bis 1927. Gleichwohl sah man sich mit gewaltigen Problemen konfrontiert, vor allem die aufgrund des sumpfigen Untergrundes notwendigen zahlreichen Änderungen - etwa die Verwendung von Eisenbetonpfählen - verschlangen deutlich mehr Geld als geplant. ${ }^{160}$ Trotz allem: Pünktlich zum 50. Verlagsjubiläum 1927 konnte Europas größtes Druckhaus den Betrieb aufnehmen. Im gleichen Jahr beschloss die Bezirksversammlung Tempelhof, die dort entlang verlaufende Burggrafenstraße in Ullsteinstraße umzubenennen.

Während das Ullstein-Imperium auf seinem vorläufigen Höhepunkt war, gab es erste Anzeichen für personelle Veränderungen an der Verlagsspitze. Hans Ullsteins ältester Sohn Karl hatte sich nach seiner Rückkehr aus französi-

156 Vgl. Ullstein, Hermann: We blundered Hitler into power. In: Saturday Evening Post, Nr. 213, 13. Juli 1940, S. 12. Aus politischen Gründen habe sich Ullstein 1931 auch von Kurt Tucholsky getrennt, so Bemmann, Helga: Kurt Tucholsky. Ein Lebensbild. Berlin 1990, S. 467 f. 157 Siehe Bannehr, Eule, S. 16.

158 Vgl. zur Baugeschichte: Braun, Immanuel [u. a.]: Ein Industriebau von der Fundierung bis zur Vollendung. Berlin 1927.

159 Siehe Bannehr, Eule, S. 17.

160 Hierzu Mendelssohn, Zeitungsstadt, S. $411 \mathrm{f}$. 
scher Kriegsgefangenschaft in den vergangenen Jahren um die Ullstein-Aktivitäten in Wien gekümmert, nun kam er zurück an die Spree: Als er 1926 in den Vorstand der Ullstein-Druckerei eintrat, leitete er sowohl die Druckerei in Tempelhof als auch jene in der Kochstraße. Überhaupt sollte nun die dritte UllsteinGeneration mehr und mehr in die Geschicke des Verlags eingreifen, was nicht ohne Zusammenstöße ablaufen sollte.

Nie zuvor und nie wieder danach wurden in Berlin so viele Zeitungen hergestellt wie in den publizistischen Boomjahren zwischen 1927 und 1929. Das Haus Ullstein hatte von den drei Berliner Verlagsimperien den größten Anteil an dieser positiven Entwicklung. Ullstein und Mosse hielten in den Weimarer Jahren zeitweilig gemeinsam einen Marktanteil von 60\%. ${ }^{161}$

Die Berliner Morgenpost war an diesem Punkt für Scherls Berliner Lokal-Anzeiger genauso uneinholbar wie auf dem Gebiet der Wochenzeitungen die Grüne Post und die Berliner Illustrirte Zeitung für den Rest des konkurrierenden Feldes. Lediglich bei den Abendausgaben konnte Scherl bis 1929 Plätze gut machen: Seiner Berliner Illustrierten Nachtausgabe konnten weder Ullsteins Tempo noch Mosses Acht-Uhr-Abendblatt ernsthaft gefährlich werden. ${ }^{162}$

Die einzige Ullstein-Publikation, die bereits 1929 einen leichten Knick in der Auflage aufwies, war die bis dahin für die Mittags-Konkurrenz unerreichbare B.Z. am Mittag (1928: 197.000 verkaufte Exemplare, 1929: 190.500 Exemplare) nicht verwunderlich, bedenkt man, dass Zeitungen im Straßenverkauf eher unter Konjunkturschwankungen leiden als jene, die zusätzlich per Abonnement vertrieben werden.

Ein anderes Problem der B.Z. am Mittag war Scherls Nachtausgabe, die zum Überholmanöver ansetzte. Doch das tat dem Ullstein-Erfolg keinen Abbruch: 1929 waren in der Kochstraße und im Druckhaus Tempelhof fast 10.000 Mitarbeiter beschäftigt, mit einem Jahresumsatz von 70 Mio. RM war Ullstein der größte Verlag Europas und gehörte zu den größten Verlagen der Welt. ${ }^{163}$

Mit Beginn der Weltwirtschaftskrise ab Oktober 1929 sanken nicht nur die Auflagenziffern der meisten Zeitungen, auch das Anzeigengeschäft wies einen deutlichen Abwärtstrend auf - was sich vor allem für die Zeitschriften als fatal erwies. Der Ullstein-Verlag verlor in dieser Zeit etwa $25 \%$ seiner Leserschaft. ${ }^{164}$ Hermann Ullstein schrieb rückblickend: „Obgleich uns die Leser nach außen

161 Siehe Fulda, Politik der „Unpolitischen“, S. 50.

162 Vgl. hierzu Gossel, Medien und Politik, S. $204 \mathrm{f}$.

163 Allein im Druckhaus Tempelhof wurden 1929 in jedem Monat über 9 Mio. Zeitschriften und Bücher gedruckt. Nach Bannehr, Eule, S. 24.

164 Hierzu Mendelssohn, Peter de: Als die Presse gefesselt war. In: Hundert Jahre Ullstein 1877-1977. Band 3. Hrsg. von Joachim W. Freyburg u. Hans Wallenberg. Frankfurt a. M./Berlin/Wien 1977. S. 193-244, hier S. 195. 
hin treu blieben, bestand kaum Zweifel, dass sie im Herzen nicht mehr bei uns waren. Die Hälfte von ihnen war bereits innerlich in Hitlers Lager angekommen. Sie waren davon überzeugt, ,dass die Dinge so nicht weitergehen können'."165

Spürbar setzte die Absatzkrise bei den meisten Ullstein-Zeitungen erst 1931 ein, bei den Morgenzeitungen früher als bei den Wochenzeitungen. ${ }^{166}$ Für die Ullstein-Zeitschriften Berliner Illustrirte und Grüne Post begann der Abwärtstrend im Juli 1931: Die Illustrirte, die noch im Oktober 1929 ca. 1,95 Mio. Stück/ Woche absetzte, fiel bis zum Januar 1933 auf 1,5 Mio. zurück; die Grüne Post verzeichnete im gleichen Zeitraum einen Rückgang von 1,25 Mio. Verkaufsexemplare auf $833.000 .{ }^{167}$

Tab. 1: Die Entwicklung der Verkaufsauflage ausgewählter Berliner Tageszeitungen zwischen 1925 und 1932 (Angaben in Tausend) ${ }^{168}$

\begin{tabular}{llllllllll}
\hline Verlag & Zeitung & 1925 & 1926 & 1927 & 1928 & 1929 & 1930 & 1931 & 1932 \\
\hline Ullstein & Berliner Morgenpost & 492 & 569 & 581.5 & 608.5 & 617 & 607.5 & 553 & 478 \\
Ullstein & Vossische Zeitung & 36 & 58 & 66.5 & 69 & 72 & 76.5 & 69 & 56 \\
Ullstein & B.Z. am Mittag & 180 & 180.5 & 186 & 197 & 190.5 & 183.5 & 167 & 151.5 \\
Ullstein & Tempo & - & - & - & - & 118.5 & 142 & 122 & 106 \\
Mosse & Berliner Tageblatt & 170 & 163 & 158 & 150 & 137 & 121 & 140 & 130 \\
Mosse & Acht-Uhr-Abendblatt & 90 & 91.5 & 93 & 95 & 97.5 & 100 & 90 & 80 \\
Mosse & Berliner Volks-Zeitung & 90 & 80 & 75 & 70 & 73 & 77.5 & 75 & 80 \\
Scherl & Berliner Lokal-Anzeiger & 220.5 & 209 & 212.5 & 219 & 219.5 & 213 & 197.5 & 183.5 \\
Scherl & Nachtausgabe & 37.5 & 59.5 & 66 & 127.5 & 193.5 & 206.5 & 197 & 185 \\
Scherl & Der Tag & 86.5 & 74.5 & 72 & 77 & 71 & 69.5 & 67.5 & 57.5 \\
\hline
\end{tabular}

Die Auflagenverluste für die Tageszeitungen des Ullstein-Verlags während der Weltwirtschaftskrise waren signifikant. Die Vossische Zeitung etwa, die noch 1929 mit 72.000 Verkaufexemplaren pro Tag ein Rekordhoch verzeichnet hatte, fiel bis 1932 wieder auf 56.000. Die Berliner Morgenpost, die noch unter nationalsozialistischer Herrschaft die mit Abstand größte Zeitung Berlins war, verlor zwischen 1929 und 1932 etwa 139.000 Verkaufsexemplare pro Tag - das ist mehr, als andere Zeitungen selbst in guten Zeiten absetzten.

Während Scherls Berliner Lokal-Anzeiger und Der Tag eine ebenfalls durch die Wirtschaftskrise bedingte kontinuierliche Abwärtsbewegung aufwiesen,

165 Nach Ullstein, Haus Ullstein, S. 206.

166 Vgl. Mendelssohn, Zeitungsstadt, S. $425 \mathrm{f}$.

167 Mendelssohn, Zeitungsstadt, S. 426 weist darauf hin, dass im Gegenzug das Ullsteinsche Blatt der Hausfrau, das freilich ohnehin vom Aktualitätsdruck und der politischen Berichterstattung befreit war, zwischen Oktober 1929 und April 1931 seine Auflage von 501.000 auf 551.000 Exemplare verbesserte.

168 Angaben der Tabelle nach Fulda, Press and Politics, S. 24. 
startete die Nachtausgabe ordentlich durch: Zwischen 1927 und 1928 vollzog sie einen Auflagensprung um etwa 93 \% auf 127.500 Verkaufsexemplare, bis 1930 setzte die Nachtausgabe 206.500 Stück/Tag ab und hatte damit ihre größte direkte Konkurrentin, die B.Z. am Mittag, überflügelt.

Wenn der Scherlschen Boulevardzeitung in den beiden Folgejahren auch die Wirtschaftskrise zusetzte, so steht sie doch für den damals aufstrebenden Typus der politisch deutlich positionierten, in diesem Fall nationalistischen, Publikation, die in einer sich polarisierenden Gesellschaft wachsenden Anhang fand. Die Nachtausgabe, die binnen weniger Jahre die Auflage des seit 1883 bestehenden Berliner Lokal-Anzeigers einholte, stellt somit den Prototyp der erfolgreichen Zeitung der Weimarer Ära der, dessen Siegeszug aus der Vermischung unternehmerischer und politischer Strategie resultierte. ${ }^{169}$ Die UllsteinProdukte strebten solche deutliche Positionierung, wie bereits dargelegt, nicht an.

Die verlags- und familieninternen Vorkommnisse der Jahre 1930/31 zwangen die Zeitungen und Zeitschriften von Europas größtem Verlag sogar noch stärker in eine andere, eine neutrale Richtung.

Da Hans und Louis, die beiden ältesten Brüder, altersbedingt kaum noch am Verlagsgeschehen teilnahmen, und Rudolf sich ganz auf den technischen Bereich konzentrierte - alles Verlegerisch-Strategische interessierte ihn kaum -, blieben nur noch zwei Brüder, die um den Vorrang im Verlag konkurrierten: Franz und Hermann. ${ }^{170}$

169 Vgl. Requate, Kommerzialisierung, S. 131, der zudem darauf hinweist, dass der für das 19. Jahrhundert geltende Gegensatz von verkaufsorientierter Presse und Gesinnungspresse in der Weimarer Republik somit obsolet wurde. Dabei zeigten sich gerade die im 19. Jahrhundert gegründeten Großverlage wie eben Ullstein, Mosse, Huck und Girardet (und damit eben jene „verkaufsorientierte“ Presse) resistent gegen industrielle Einflussnahmen. Requate, Kommerzialisierung, S. 135: „Will man diese Zahlen [die Auflagenzahlen der Tagespresse der Weimarer Ära, d. Verf.] interpretieren, ist offensichtlich, dass auch hier kein einfacher Gegensatz von politischer Orientierung und Verkauforientierung auszumachen ist. Vielmehr bedienten sich diejenigen, die klare politische Ziele mit ihren Zeitungen verfolgten, wie Hugenberg für die DNVP, Münzenberg für die KPD und schließlich auch die NSDAP mit dem ,Angriff‘, der Stilmittel moderner Boulevardzeitungen, um ihre potenzielle Anhängerschaft auszuschöpfen und so eine Symbiose aus Käufer und Wähler herzustellen.“ Willi Münzenberg etwa hatte es geschafft, die Auflage der sozialistischen Boulevardzeitung Welt am Abend zwischen 1925 und 1928 von 12.000 auf 185.000 Verkaufsexemplare zu steigern.

170 Hermann Ullstein: „So blieb es an Franz und mir zu rivalisieren. Für ihn ging es um absoluten Führungsanspruch, für mich um Gleichheit. Als älterer Bruder war er Chef, ich war Vizechef. Wir teilten die gleichen politischen und verlegerischen Ansichten. Er war der Leiter der Zeitungsabteilung, ich war für Zeitschriften und später auch für die Buchproduktion zuständig. Franz war für eine strikte Zentralisierung der Macht, wohingegen ich der Dezentralisierung den 
Diese Rivalität hatte, wie bereits geschildert, etwa im Streit um die politische Ausrichtung der Ullstein AG ihren Widerhall gefunden. Hermann entschied schließlich, sich nur noch um seine Neue Leipziger Zeitung zu kümmern. Zurück blieben der als diktatorisch verschriene Franz als Chef des Hauses und die nunmehr dritte Ullstein-Generation, die jetzt auf Mitsprache drängte. Mitten in der schlimmsten wirtschaftlichen und politischen Krise, die Deutschland bis dahin erlebt hatte, waren die Ullsteins mit sich selbst beschäftigt.

Georg Bernhard, der Chefredakteur der Vossischen Zeitung, war seit 1924 Mitglied der DDP, vier Jahre später saß er für die Partei auch im Reichstag. Er bot damit nun verlagsintern gleich mehrere Angriffsflächen: Aufgrund seiner Abgeordnetentätigkeit wurde seine Anwesenheit in der Redaktion lückenhafter; Bernhards explizitere politische Ausrichtung der Vossischen Zeitung passte nicht zu der bei Ullstein verfolgten Linie der Neutralität; seine seit Jahren offen dargelegte Frankophilie machte ihn zum Objekt der rechten Presse. Und schließlich fuhr die altehrwürdige „Tante Voss“ bei allem Renommee noch immer jedes Jahr siebenstellige Verluste ein, allein 1928 ca. 2 Mio. RM. ${ }^{171}$ Noch aber stand Georg Bernhard unter der Protektion des Vorstandsvorsitzenden Franz Ullstein.

Dies änderte sich am Jahresende 1929, als Franz die deutlich jüngere Journalistin Dr. Rosie von Gräfenberg heiratete. Bei den Ullsteins brach „Panik“ vor der dynamischen jungen Frau aus, wie Franz Ullstein später, nach verlorenem Kampf, schilderte. ${ }^{172}$ Vier der fünf Brüder fürchteten, dass Rosie die Alleinherrschaft Franz Ullsteins zum Schaden der auf Mitsprache drängenden dritten Generation verlängern könnte. Georg Bernhard war ebenfalls besorgt, nämlich dass die Gräfin ihren Günstling, den konservativen Friedrich Sieburg, nun zum Chefredakteur der Vossischen protegieren könnte. Bernhard warf also Rosie öffentlich „Gerissenheit“ vor. ${ }^{173}$ Im Verlag bildeten sich zwei scheinbar unversöhnlich gegenüberstehende Lager. ${ }^{174}$ Politisch wurde der familien- und verlagsinterne Konflikt, als die französische Wochenzeitschrift Aux Ecoutes am

Vorzug gab. So hatte ich etwa dafür gesorgt, dass im Vorstand alle Mitglieder gleiches Stimmrecht besaßen." Nach Ullstein, Haus Ullstein, S. $208 \mathrm{f}$.

171 Angaben nach Fulda, Press and Politics, S. 30.

172 So Bornstein, Josef: Ullstein-Roman. In: Das Tagebuch 11 (1930), Heft 26, S. 1020-1033.

173 Über den Schlagabtausch siehe Koszyk, Deutsche Presse 1914-1945, S. 253 f. In der anstehenden Darstellung wird vor allem Koszyks Schilderung gefolgt.

174 Arthur Koestler erinnerte sich: „Die Anschuldigung wurde schließlich in einem langen, schmutzigen Prozess widerlegt; doch spaltete er die Firma, vom Chefredakteur bis zum Botenjungen, in zwei feindliche Lager: die ,Franziskaner', die zu Dr. Franz Ullstein hielten, und die ,Bernhardiner', die ihren Namen vom Chefredakteur der Vossischen Zeitung, Georg Bernhard, ableiteten.“ Aus: Koestler, Arthur: Frühe Empörung. Autobiographische Schriften. Band 1. Berlin/München 1993, S. 209. 
30. November 1929 berichtete, dass Gräfin Rosie während ihres Paris-Aufenthaltes in den 1920er Jahren als Agentin des deutschen Botschafters gearbeitet habe. Nationalistische deutsche Zeitungen machten nun aus ihr eine französische Spionin. Die Gerüchte hatte Josef Matthes, ein ehemaliger Separatistenführer, verbreitet.

Im Januar 1930 nahmen Louis und Rudolf Ullstein Kontakt mit Matthes in Paris auf, der ihnen Kopien angeblicher Beweise zukommen ließ. Offenbar hatten die Vorbehalte gegen Franz, den „Diktator“, in der Familie überhandgenommen. Die angeblich belastenden Kopien wurden zwar Georg Bernhard, nicht aber dem gerade von der Hochzeitsreise zurückkehrenden Franz Ullstein vorgelegt. Seine Brüder stellten Franz am 18. Januar 1930 vor die Wahl: entweder Scheidung oder Karriereende. Zehn Tage später wurde Dr. Franz Ullstein aus dem Vorstand abberufen. Franz und seine Kinder Elisabeth (später, in der Emigration, schrieb sie sich „Elizabeth“) und Kurt waren fortan isoliert. Diese Ausgrenzung des Stammes Franz sollte bei der Restitution der Ullstein AG nach dem Krieg eine entscheidende Rolle spielen.

Das Ehepaar Franz und Rosie Ullstein erhob nun Klage auf Widerruf der Spionage-Gerüchte, u. a. gegen Louis und Rudolf Ullstein sowie gegen Georg Bernhard (der mit dem Ausscheiden Franz Ullsteins seinen einst wichtigsten Befürworter verloren hatte). Vor dem Landgericht I in Berlin begann der Prozess am 1. Mai 1930. Louis und Rudolf behaupteten, neues belastendes Material über Rosie und ihre angeblichen „revolutionären Umtriebe“ $\mathrm{zu}$ haben, Reichswehrminister von Schleicher ließ sicherheitshalber rasch mitteilen, dass überhaupt kein Material über die Gräfin vorhanden sei.

Während das Verfahren sich in den folgenden Monaten hinzog, einigten sich Georg Bernhard und der Ullstein-Verlag über ein Ausscheiden Bernhards zum Jahresende. Auch der Reichsverband der Deutschen Presse, deren Vorsitzender er war, und die DDP befassten sich mit Bernhard: Ein parteiinternes Ehrengericht, dem u. a. Otto Nuschke und Theodor Heuss angehörten, befand ihn schuldig, Fehler begangen zu haben, die mit seiner Stellung als Publizist und Politiker nicht zu vereinbaren seien. Für die nun gebildete Staatspartei wurde Georg Bernhard bei den anstehenden Wahlen im September 1930 nicht mehr aufgestellt. ${ }^{175}$ Im Oktober trat Georg Bernhard, der auch durch den von ihm geführten Reichsverband der deutschen Presse keine Verteidigung erfuhr, von dessen Vorsitz zurück.

Franz Ullstein wiederum revanchierte sich publizistisch: Er beauftragte den Schriftsteller Josef Bornstein mit dem Verfassen einer Denkschrift gegen Georg

175 Hauptkritikpunkt waren Bernhards Kontakte zum Separatistenführer Matthes, die zu einer Belastung für die Partei wurden. Koszyk, Deutsche Presse 1914-1945, S. 255. 
Bernhard, ein Auszug erschien 1930 in der Zeitschrift Das Tagebuch unter dem Titel Ullstein-Roman. Darin wird Franz Ullsteins Meinung über das Grundproblem der ganzen Auseinandersetzung deutlich, das für ihn in der dritten Ullstein-Generation lag, die nun im Verlag an die Macht wollte: „Die Zeitung kann sich nicht, wie ein Seifengeschäft oder eine Gastwirtschaft, einfach auf den leiblichen Nachwuchs vererben. Dazu ist ihre Funktion im geistigen Leben der Nation zu bedeutsam. [...] Große Zeitungen müssen vor den Kläglichkeiten eines Familienstreites und der Torschlusspanik von Politikern geschützt werden, über die die Zeit hinwegzuschreiten droht.“176

Georg Bernhard nahm Auszüge aus dem Ullstein-Roman zum Anlass zu einer Gegenklage wegen Beleidigung gegen Franz Ullstein und Josef Bornstein. Der Prozess, am 19. März 1931 beim Amtsgericht Berlin-Mitte eröffnet, endete zehn Tage später mit einem Freispruch. ${ }^{177}$ Im Februar 1933 zwangen die Nationalsozialisten Georg Bernhard, Deutschland zu verlassen, am 25. August 1934 wurde ihm die Staatsangehörigkeit aberkannt. Im Exil arbeitet er am Pariser Tageblatt mit, nach seiner Flucht 1941 in die USA verstarb er dort im Jahr 1944 in einem Hospiz in Harlem. ${ }^{178}$

Louis' Sohn Heinz ging als neuer Chef der Tageszeitungssparte als größter Gewinner aus dem Familienkonflikt hervor. Auch Hans' Schwiegersohn Fritz Ross, der seit 1924 Leiter der Zeitschriftensparte war, stieg nun in den Aufsichtsrat auf, während sein Schwager Karl für die Druckereien in Tempelhof und der Kochstraße verantwortlich blieb.

Zumindest der unmittelbare Anlass für den Ausbruch der Familienfehde war beseitigt, denn Franz hatte sich von Rosie im Oktober 1930 scheiden lassen. Am 12. März 1931 begann der Prozess Franz und Rosie Ullstein gegen Hans, Louis und Rudolf Ullstein (Hermann hatte sich komplett herausgehalten). Staatssekretär Hans Schäffer aus dem Finanzministerium wurde mit der Leitung eines Schiedsgerichts betraut, im Juni 1931 konnte er einen Vergleich herbeiführen. ${ }^{179}$

176 Bei Koszyk, Deutsche Presse 1914-1945, S. 254.

177 Den Beklagten billigten die Richter sogar etwas Wiedergutmachung zu. So erklärten die Brüder Ullstein am 31.03.1931 in der Vossischen Zeitung: „Wir fühlen uns [...] verpflichtet, unserer Überzeugung dahin Ausdruck zu geben, dass Frau Dr. Rosie Ullstein sich nicht als Spionin betätigt hat, und dass auch ein gerechtfertigter Verdacht der Spionage nicht besteht." Bei Koszyk, Deutsche Presse 1914-1945, S. 255.

178 Mehr über Intellektuellenschicksale im Exil bei: Groth, Michael: The Road to New York. The Emigration of Berlin Journalists 1933-1945. München/New York/London/Paris 1988.

179 Schäffer erwarb sich hierdurch das Vertrauen der Ullsteins. Im Juni 1932 berief man ihn als Generaldirektor in die Verlagsleitung. Er konnte sogar an den zweimal wöchentlich stattfindenden „politischen Besprechungen“ der Chefredakteure teilnehmen - hier wurde die politische Linie der Ullstein-Publikationen koordiniert. Siehe Wandel, Eckhard: Hans Schäffer. Steuermann in wirtschaftlichen und politischen Krisen. Stuttgart 1974, S. 236. 
Die Ullsteins erklärten daraufhin offiziell ihre Versöhnung. ${ }^{180}$ Franz war nun kein Generaldirektor mehr, aber er und Hermann erhielten wieder Büros in der Kochstraße und nahmen an den Aufsichtsratssitzungen teil. Die dritte UllsteinGeneration hatte den Kampf um die Macht für sich entschieden.

Der große Streit der Jahre 1930/31, der eine desaströse Wirkung nach innen und in der Öffentlichkeit entfaltete ${ }^{181}$, weist auf den Grad der Entfremdung hin, den die mittlerweile kaum noch überschaubare Familie anlässlich des Eintretens der „Enkel-Generation“ in den Verlag erfahren hat. Eine Entfremdung, die auch bei den Vorbereitungen der Restitution nach dem Zweiten Weltkrieg deutlich hemmende Auswirkungen bei der Kooperation zwischen den fünf UllsteinStämmen haben wird. Die Ullsteins mussten aber nun, im Jahr 1931, rein aus pragmatischen Gründen und zumindest nach außen hin geschlossene Reihen bilden.

Noch am 23. September 1930 hatte sich Reichskanzler Brüning über Ullstein-Blätter in der Kabinettssitzung beschwert, Anlass waren Berichte in der B.Z. am Mittag über angebliche Putschvorbereitungen der Nationalsozialisten. ${ }^{182} \mathrm{Zu}$ Beunruhigungen dieser Art gaben die Ullstein-Blätter unter der neuen Verlagsführung nun keinen Anlass mehr. ${ }^{183}$ Mit der am 6. Oktober 1931 beschlossenen Notverordnung schuf das Kabinett Brüning ohnehin die Voraussetzungen, um unliebsam in Erscheinung getretene Zeitungen durch die Androhung von Landesverratsverfahren gefügig zu machen.

Diese Verordnung war eine von neun zwischen dem 28. März 1931 und dem 19. Dezember 1932 erlassenen Präsidialverordnungen, mit denen zunächst Reichskanzler Brüning und nach ihm Franz von Papen der um sich greifenden Radikalisierung Herr zu werden versuchten; die meisten dieser Bestimmungen dienten der Eindämmung militanter Ausbrüche, einige jedoch waren auch geeignet, Druckschriften zu verbieten oder Journalisten strafrechtlich verfolgen $\mathrm{zu}$ lassen: So wurden allein 1931 insgesamt 224, ein Jahr später sogar 294 Zeitungen zeitweilig verboten. ${ }^{184}$ Die neue Ullstein-Verlagsleitung hatte Reichskanzler Brüning 1931 zugesichert, die präsidialen Notverordnungen in ihrer Berichterstattung zu unterstützen. ${ }^{185}$

Von den drei großen Berliner Verlagshäusern traf die Weltwirtschaftskrise Mosse am härtesten: Das von Theodor Wolff geleitete Berliner Tageblatt, das Flaggschiff des Verlags, litt genauso unter der schwindenden Auflagenentwick-

180 Vgl. Nadolny, Ullsteinroman, S. 451.

181 Dazu Schütz, Antlitz, S. 22.

182 Siehe B.Z. am Mittag vom 18. und 19. September 1930.

183 Hierzu Koszyk, Deutsche Presse 1914-1945, S. 256.

184 Vgl. Gossel, Medien und Politik, S. 183.

185 Nach Bannehr, Eule, S. 43. 
lung wie die anderen Berliner Zeitungen, teure finanzielle Manöver wie der Ankauf des Acht-Uhr-Abendblatts schlugen nun umso deutlicher zu Buche.

Am schlimmsten aber wirkte sich die negative volkswirtschaftliche Entwicklung auf das eigentliche Rückgrat Mosses, die Anzeigenagentur, aus. ${ }^{186}$ Der Einbruch des Anzeigengeschäfts und die starke Konkurrenz durch Hugenbergs Allgemeine Anzeigen GmbH belasteten den Verlag zu sehr: Im September 1932 musste Mosse Konkurs anmelden.

Seit 1929 gingen auch die Gewinne des Ullstein-Verlags zurück: War 1928 ein Reingewinn von 1,9 Mio. RM erwirtschaftet worden, so lag er 1931 bei nur noch 629.000 RM. ${ }^{187}$ Neben den wirtschaftlichen Umständen führte jedoch noch ein weiterer nicht unwesentlicher Faktor zu den Verlusten bei Auflage und Anzeigenaufkommen: Aufsichtsratsmitglied Fritz Ross, Schwiegersohn von Hans Ullstein, vermerkte die spürbaren Auswirkungen der Boykottaufrufe der Nationalsozialisten gegen Ullstein-Produkte. ${ }^{188}$ Auch die Banken gaben sich nun gegenüber dem Verlagshaus zurückhaltender, vor allem ab $1932 .{ }^{189}$

Aufgrund dieser Einbußen kam es nun auch zu Entlassungen (der Personalabbau betrug zwischen 1930 und 1932 weniger als $5 \%$ ) und erstmals zu ,wilden Streiks“ im Druckhaus Tempelhof - schließlich bedeuteten dünnere Zeitungen auch weniger Arbeit für die Setzer und Drucker. ${ }^{190}$

$186 \mathrm{Zu}$ den Folgen für Mosse auch Gossel, Medien und Politik, S. 204.

187 Angaben nach der Aufstellung „Wie der Aktienbesitz der Familie Ullstein an den Parteiverlag überging“ des Verlags-Buchhalters Gustav Willner, datiert auf den 02.08.1945, in: ASUA, Bestand Ullstein, Band 4.

188 „Die blind folgenden Parteimitglieder wurden in den Naziblättern zum Boykott aller Verlagsprodukte des Ullstein-Verlages aufgefordert, sowie zum Boykott jeder Inserierung. [...] Wenn auch die Masse der Leser und Inserenten den Blättern des Ullsteinhauses die Treue hielt, so verursachten doch die dauernden Angriffe der Nazis zu Beginn der 30er Jahre eine empfindliche Einbuße seiner Einnahmen.“ Aussage von Fritz Ross vom 31.07.1951, in: AS-UA, Bestand Ullstein, Band 17.

189 In einer eidesstattlichen Versicherung, datiert auf den 01.12.1945, erklärte Gustav Willner, der Leiter der Ullstein-Buchhaltung: „Die Auswirkungen der Nazi-Propaganda hatten nicht den Erfolg, den die Partei erwartete, sie erreichten aber immerhin, dass die Kreditgebenden Banken vorsichtig wurden, und namentlich die Reichs-Kredit-Gesellschaft brachte in den Besprechungen ganz klar zum Ausdruck, dass sie allergrößten Wert darauf lege, dass von den in Anspruch genommenen Krediten nicht etwa die Mitglieder der Familie - sei es in Form von Gehältern oder Spesen - irgendwelche Beträge erhielten. Es gelang damals, weil die beiden anderen Banken zu der Geschäftsleitung Vertrauen hatten, die Reichs-Kredit-Gesellschaft überhaupt aus den Verhandlungen auszuschalten und die finanzielle Lage der Firma durch Zusagen der Deutschen Bank und der Berliner Handelgesellschaft wieder zu sichern.“ Aussage Willners vom 01.12.1945 in: AS-UA, Bestand Ullstein, Band 17.

190 Ein Zeitzeuge erinnert sich bei Bannehr, Eule, S. 28, über entsprechende Vorkommnisse aus dem Jahr 1929: „Die ,Berliner Illustrirte‘ hatte normalerweise 48 Seiten: Hatte sie 40, dann 
Dennoch stand das Haus an der Kochstraße bedeutend besser da als seine schwankenden oder gefallenen Konkurrenten, noch immer produzierte Ullstein 350 Mio. Zeitungen und 115 Mio. Zeitschriften pro Jahr.

brauchten sie ein Werk weniger. Jedes Werk hat 16 Seiten gehabt, dann brauchten sie schon einen Drucker weniger. Manchmal haben wir auch bloß 32 Seiten gedruckt, dann brauchten sie noch einen Drucker weniger. Dann wurden welche entlassen, die später angefangen hatten. Und wenn wieder welche gebraucht wurden, dann wurden wieder welche eingestellt. Damit die Kollegen, die arbeitslos waren, keine Lohndrücker machten, haben wir extra Geld gesammelt, damit sie ein gewisses Geld von uns bekamen, die wir noch Arbeit hatten. [...] Wir haben ja auch manchmal die Maschinen stehenlassen in der ,Illustrirten', hat drei Stunden gedauert...“. Bannehr thematisiert zudem die Arbeitsaussetzungen der Rotationshilfsarbeiter um den 8. und 9. Januar 1932, die zu Notausgaben u. a. von Tempo und Vossischer Zeitung führten (Bannehr, Eule, S. 31). 
